\title{
Energetic electron detection packages on board Chinese navigation satellites in MEO
}

\author{
YuGuang Ye1, Hong Zou ${ }^{1}$, Qiu-Gang Zong ${ }^{1}$, HongFei Chen ${ }^{1}$, JiQing Zou ${ }^{1}$, WeiHong Shi ${ }^{1}$, XiangQian Yu ${ }^{1}$, \\ WeiYing Zhong ${ }^{1}$, YongFu Wang' ${ }^{1}$, YiXin Hao' , ZhiYang Liu ${ }^{1}$, XiangHong Jia ${ }^{2}$, Bo Wang ${ }^{3}$, \\ XiaoPing Yang ${ }^{3}$, and XiaoYun $\mathrm{Hao}^{3}$ \\ ${ }^{1}$ School of Earth and Space Sciences, Peking University, Beijing 100871, China; \\ 2State Key Laboratory of Space Medicine Fundamentals and Application, Chinese Astronaut Research and Training Center, Beijing 100093, China; \\ ${ }^{3}$ Shandong Aerospace Electro-technology, Yantai Shandong 264000, China
}

Key Points:

- Energetic electron detection packages (EEDP) were deployed on three Chinese navigation satellites in medium Earth orbit (MEO).

- The ground calibration and preliminary observations verified the good performance of the instruments.

- The results of EEDPs provide important support for theoretical research on the radiation belts and the applications related to space weather.

Citation: Ye, Y. G., Zou, H., Zong, Q.-G., Chen, H. F., Zou, J. Q., Shi, W. H., Yu, X. Q., Zhong, W. Y., Wang, Y. F., Hao, Y. X., Liu, Z. Y., Jia, X. H., Wang, B., Yang, X. P. and Hao, X. Y. (2021). Energetic electron detection packages on board Chinese navigation satellites in MEO. Earth Planet. Phys., 5(2), 158-179. http://doi.org/10.26464/epp2021021

\begin{abstract}
Energetic electron measurements and spacecraft charging are of great significance for theoretical research in space physics and space weather applications. In this paper, the energetic electron detection package (EEDP) deployed on three Chinese navigation satellites in medium Earth orbit (MEO) is reviewed. The instrument was developed by the space science payload team led by Peking University. The EEDP includes a pinhole medium-energy electron spectrometer (MES), a high-energy electron detector (HED) based on $\triangle \mathrm{E}-\mathrm{E}$ telescope technology, and a deep dielectric charging monitor (DDCM). The MES measures the energy spectra of 50-600 keV electrons from nine directions with a $180^{\circ} \times 30^{\circ}$ field of view (FOV). The HED measures the energy spectrum of $0.5-3.0 \mathrm{MeV}$ electrons from one direction with a $30^{\circ}$ cone-angle FOV. The ground test and calibration results indicate that these three sensors exhibit excellent performance. Preliminary observations show that the electron spectra measured by the MES and HED are in good agreement with the results from the magnetic electron-ion spectrometer (MagEIS) of the Van Allen Probes spacecraft, with an average relative deviation of $27.3 \%$ for the energy spectra. The charging currents and voltages measured by the DDCM during storms are consistent with the highenergy electron observations of the HED, demonstrating the effectiveness of the DDCM. The observations of the EEDP on board the three MEO satellites can provide important support for theoretical research on the radiation belts and the applications related to space weather.
\end{abstract}

Keywords: radiation belts; energetic electron detection; Pin-hole technology; Chinese navigation satellites; MEO; internal charging

\section{Introduction}

The region from $2000 \mathrm{~km}$ ( $L$-shell $\sim 1.3$ ) to $\sim 25,000 \mathrm{~km}(L \sim 5)$ above the ground is called medium Earth orbit (MEO) to distinguish it from low Earth orbit (LEO, $L<1.3$ ) and geostationary Earth orbit (GEO, $L \sim 7)$. MEO is located in the center of the outer radiation belt where relativistic electron radiation is the highest, and the electron flux is several times to one order of magnitude higher than in GEO.

The high flux of relativistic electrons causes internal charging (IC)

Correspondence to: H. Zou, hongzou@pku.edu.cn

Received 24 NOV 2020; Accepted 26 JAN 2021.

Accepted article online 01 MAR 2021.

(C)2021 by Earth and Planetary Physics. and discharging of spacecraft, leading to satellite failures and heavy losses (Leach and Alexander, 1995). The orbits of most satellites (including scientific satellites) are located in LEO and GEO to prevent radiation damage; thus, most scientific observations are obtained in these two regions. To date, our understanding of IC in the international space community is primarily derived from satellite incidents in GEO. However, the relative electron flux intensity is much higher and IC is more likely to occur in MEO than in GEO.

Before the development of the Van Allen Probes (VAP), the most well-known satellite mission in the MEO region was the Combined Release and Radiation Effects Satellite (CRRES). This mission is recognized not only for its primary observations in the MEO outer belt radiation zone, but also due to the impact of high-flux electron radiation on the satellite. In July 1990, CRRES was launched 
into a geostationary transfer orbit close to the equator $(L \sim 2.5-6.7$ with its main goal to improve understanding of the effects of the space radiation environment (Johnson and Kierein, 1992). Soon after launch, the CRRES instruments encountered a high-flux energetic electron zone that quickly appeared in the slot region. This electron zone was initiated by the impact of solar wind-driven shock waves on the magnetosphere and lasted for several months (Blake et al., 1992). As a result, the engineering payload aboard CRRES observed IC events many times (Frederickson et al., 1991, 1992; Violet and Frederickson, 1993; Frederickson, 1996), providing space environment researchers with a better understanding of IC causes (Fennell et al., 2000). Since CRRES was being bombarded by high-energy electrons in MEO for long periods, it stopped working due to battery damage only 14 months after launch, far shorter than the designed three-year lifetime. Additionally, global positioning system (GPS) satellites also operate in MEO but their energetic electron detection payloads on board is relatively simple with low energy resolution. Since no pitch angle measurements are made, the data have not been widely used (Baker et al., 2013).

The 2012 launch of the twin VAP spacecraft (previously called Radiation Belt Storm Probes) started a new era of radiation belt detection. They were placed in two nearly identical geostationary transfer orbits with an inclination of $\sim 10^{\circ}$, a perigee of $\sim 600 \mathrm{~km}$, an apogee of $\sim 33000 \mathrm{~km}$ or $L=6$, an orbit period of 9 hours, and variable spacecraft separation ( $\mathrm{Li}$ and Hudson, 2019). VAP was equipped with comprehensive particle, magnetic and electric field, and wave detection payloads, which obtained detailed measurements during the maximum and declining phases of solar cycle 24 (Mauk et al., 2013). VAP observations have enhanced our understanding of the acceleration, transmission, and loss mechanisms of energetic electrons in the radiation belts (Baker et al., 2018, 2019; Li and Hudson, 2019, and the references therein).

Baker et al. (2013b) reported several MeV electrons measured by the Relativistic Electron-Proton Telescope (REPT) (Baker et al., 2013a) and discovered the three-belt structure of the radiation belt. Hao YX et al. (2020) conducted energetic electron measurements using the magnetic electron-ion spectrometer (MagEIS) and found that electrons with an energy of several hundred keV also formed a three-belt distribution. This triple structure is related to the generation and loss mechanism of high-energy electrons in the outer radiation belt (Mann et al., 2016). High-energy electrons generated in a previous event were affected by an electron loss mechanism in the outer radiation belt driven mainly by the magnetopause shadowing effect. In some cases, the loss mechanism only significantly impacted electrons in the higher $L$ shell region of the outer radiation belt, whereas high-energy electrons in the low $L$-shell region were retained to form a storage ring in the third belt (Baker et al., 2013b). Subsequently, a new highenergy electron belt was generated in the high $L$-shell region, forming a three-belt structure.

Another important observation made during the VAP era is that an impenetrable barrier for hgih-energy electrons exists at the inner boundary of the outer belt (Baker et al., 2018). If high dynamic pressure, extremely strong solar wind impacts the magnetosphere, the inner boundary of the electrons in the outer radiation belt can be transmitted inward to the region $L<2$. The slot region is thus filled with electrons from several hundred keV up to 10 $\mathrm{MeV}$, and this so-called slot-penetrating event can last from several weeks to months (Selesnick et al., 2016). Well-known slot-penetrating events observed before the launch of VAP included the new radiation belt event observed by CRRES in 1991 (Blake et al., 1992) and the Halloween event observed by the Solar, Anomalous and Magnetospheric Particle Explorer (SAMPEX) in late 2003 (Baker et al., 2004). This high-energy electron belt that exists for extended periods in the slot region is a major threat to satellites operating in LEO, thus, the space weather community is very concerned with this phenomenon (Fennell et al., 2000). During the VAP mission, ECT/MagEIS observed a slot-penetrating event with electrons $\leq 200 \mathrm{keV}$ during a geomagnetic storm in March 2013; however, electrons $\geq 894 \mathrm{keV}$ did not exhibit the same phenomenon (Fennell et al., 2015). The observations of the VAP REPT instrument from 2012 to 2016 indicated that the inner boundary of $>1 \mathrm{MeV}$ electrons in the outer belt did not reach $L<2.5$, which is the so-called 'impenetrable barrier' (Baker et al., 2018). It is unclear whether the impenetrable barrier is the result of physical mechanisms or occurs because the coronal mass ejection (CME) shock wave is not strong enough, preventing VAP from observing the penetration of high-energy electrons into the deeper slot region.

VAP observations not only enabled better understanding and simulation of the dynamic physical processes controlling Earth's radiation belts, but also revealed many new characteristics. Quasi-linear theory has played an important role in studying the acceleration, transmission, and loss of electrons in the outer belt. However, this theory has difficulty explaining the rapid acceleration and precipitation of electrons in the outer belt and simulating the radial diffusion driven by ULF waves, which is necessary to introduce nonlinear wave-particle interactions ( $\mathrm{Li}$ and Hudson, 2019, and the references therein). High-quality observations of electron energy spectra and pitch angle distributions from tens of $\mathrm{keV}$ to several MeV are essential for investigating nonlinear waveparticle interactions in the acceleration, transmission, and loss of outer belt electrons. With the decommissioning of VAP in 2019, new missions are needed to observe and reveal the mysteries of the radiation belts.

The simulation of dynamic outer belt processes requires high-precision observations of the inner and outer boundary conditions. The VAP orbit reached a maximum $L \sim 6$, which does not cover the outer boundary of the outer belt, therefore, radiation belt modeling requires high-quality observations encompassing a larger range (including the core and large $L$-shell regions). In addition, radiation belt modeling based on novel machine learning technology is also in need of high-quality observations of the outer belt electrons (Bortnik et al., 2018).

Due to the importance and increased applications of GPS, the $M E O$ radiation environment is receiving increased attention. With the arrival of another active solar cycle, the space science and aerospace engineering communities have reached a consensus that there is a critical need for continuous observations of the radiation environment in MEO, with a large energy range and highresolution energy spectra and pitch angle distributions. 
This article introduces the energetic electron detection package (EEDP) developed by Peking University, deployed on three Chinese navigation satellites in MEO. They can cover the core and large $L$-shell regions of the outer radiation belt, providing a convenient platform for the observation of outer belt electrons. The package includes three sensor heads: a medium-energy electron spectrometer (MES), a high-energy electron detector (HED) and a deep dielectric charging monitor (DDCM). The MES and HED can measure energy spectra from $50 \mathrm{keV}$ to $3.0 \mathrm{MeV}$, which covers the energy range of seed electrons and relativistic electrons.

Currently, there are three types of instruments that can measure medium-energy electrons. The first type is a telescope system composed of a collimator and single or multiple silicon detectors, such as the directional electron detector of the medium-energy proton electron detector (MEPED) on NOAA-POES satellites (Evans and Greer, 2004) and the low-energy particle (LEP) detector on GPS satellites (Tuszewski et al., 2004). This kind of detector has a relatively simple sensor head that can only measure electrons incident in one direction. The second detector type is a magnetic spectrometer, such as the magnetic electron and ion spectrometer (MagEIS) of VAP (Blake et al., 2013), which uses a permanent magnet to deflect electrons of different energies to silicon detectors at different positions. In order to ensure that the measured energy is not affected by the incident angle, the FOV of a magnetic spectrometer is usually very small (e.g., MagEIS is $10^{\circ} \times 20^{\circ}$ ). The third detector type is the medium-energy electron imaging spectrometer, based on the pinhole imaging technique, such as the imaging electron spectrometers (IES) on board Polar and Cluster (Blake et al., 1995; Wilken et al., 1997). The EEDP MES uses this pinhole imaging technique based on the IES sensor design of the Cluster II mission (Wilken et al., 1997), and was developed by Peking University. The first pinhole IES in China is named as BeiDa-IES (BD-IES) (Zou H et al., 2018; Zong QG et al., 2018). The BD-IES was successfully launched into an inclined geosynchronous orbit (IGSO) in September 2015 and is still in operation. Among its numerous scientific achievements are observations of substorm electron injection, wave-particle interactions, and the electron response to geomagnetic storms (Zong QG et al., 2016; Li L et al., 2017a, b; Wang LH et al., 2017; Liu ZY et al., 2018, 2019; Yin ZF et al., 2019; Chen XR et al., 2020a, b). The related sensor head of the EEDP MES can measure 50-600 keV electrons incident from nine directions within a view angle of $180^{\circ}$, from which pitch angle distributions can be derived.

The $\Delta \mathrm{E}-\mathrm{E}$ telescope detector is generally used for high-energy electron detection, using a thin silicon wafer as the $\Delta \mathrm{E}$-detector at the front of the telescope. By determining the difference in the deposited energy of the incident electrons and protons at the thin detector, the sensor can distinguish whether electrons or heavy ions have passed through the $\Delta \mathrm{E}$-detector so proton contamination in the electron measurement can be removed. The E-detector is composed of a thick silicon to measure the energy of incident electrons, with the thickness constraining the highest energy of electrons that can be measured. However, as the electron energy increases, the thickness of the shielding layer outside the telescope must also be increased to block contamination by highenergy electrons from the side of the telescope, and only meas- ure incident electrons from the telescope FOV. The VAP REPT instrument is representative of this type of high-energy electron detector, using $24 \mathrm{~mm}$ thick silicon which can measure electrons $>18.9 \mathrm{MeV}$ (Baker et al., 2013a). Its lateral shielding shell can prevent $<20 \mathrm{MeV}$ electrons from entering the $E$ detector, resulting in a sensor head weight as high as $6.5 \mathrm{~kg}$.

The High Spectrometer of MagEIS can also measure high-energy electrons, up to $4.8 \mathrm{MeV}$ using a $4800 \mathrm{G}$ permanent magnet to deflect the incident electrons to the $\Delta \mathrm{E}-\mathrm{E}$ telescope detectors and measure their energy. The HED of the EEDP employs the $\Delta \mathrm{E}-\mathrm{E}$ telescope technique with a $5 \mathrm{~mm}$ thick $E$ detector, that capable of measuring high-energy electrons from 0.5 to $3.0 \mathrm{MeV}$. Measurements using a similar sensor head structure have been verified in orbit by the high-energy particle detectors on board the ChinaBrazil Earth Resource Satellite (CBERS) and the Feng Yun Satellites (FY) (Xiao Z et al., 2003; Wang CQ et al., 2017).

The EEDPs on the three Chinese navigation satellites in MEO can provide multi-point observations of the core and high L-shell regions of the outer radiation belt, which is of great significance for studying the physical processes of electron dynamics, radiation belt modeling, and space weather applications. The next section describes the principles and characteristics of the instruments. The third section introduces the ground test and calibration results. The fourth section introduces the preliminary observations of the EEDPs on board the three MEO satellites, followed by the discussion and summary.

\section{Instrument Description}

The EEDP on the Chinese navigation satellites in MEO was developed by the instrument team at Peking University and include three types of detectors: the medium-energy electron spectrometer (MES), the high-energy electron detector (HED), and the deep dielectric charging monitor (DDCM). Their purpose is to measure the energy spectra and flux changes of medium-and high-energy electrons in the outer radiation belt, as well as the resulting deep dielectric charging current and voltage. The working principles and components of the instruments are introduced below.

\subsection{Medium-energy Electron Spectrometer (MES)}

The schematic diagram and a photo of the MES sensor head are shown in Figure 1.

Each sensor head unit is encased in a shielding box, with a pinhole on one side (Figure 1a). Inside the housing is a three-pixel segmented Si-PIN position-sensitive detector, fabricated by Micron Semiconductor Limited in the United Kingdom. Each detector pixel together with the pinhole forms a telescope covering a $20^{\circ}$ FOV. Each sensor head unit covers a $\sim 60^{\circ} \mathrm{FOV}$, with a total $180^{\circ}$ FOV covered by the three sensor head units, shown in Figure $1 \mathrm{~b}$. This type of sensor was used in the Cluster-II mission which is still operating successfully after 20 years. To minimize power and size, an integrated pre-amplifier (PA) application-specific integrated circuit (ASIC) chip processes the weak signals from the sensor head. The readout electronics for nuclear applications (RENA-3) is a low-noise integrated PA with 36 channels. Each sig- 
nal channel consists of a detector pixel and an amplifier channel of the ASIC. The sensor head consists of three three-pixel detectors, for a total of nine detector pixels, corresponding to nine independent channels of the RENA-3 chip to measure incident electrons in nine directions simultaneously.

Figure 2 shows the block diagram of the MES, consisting of the sensor head and the electronics box. The two circuit modules in the electronics box are the signal conditioning unit (SCU) and the power supply unit (PWR). The functions of the SCU are two-fold: 1) to provide the timing signals of particle event sampling to the ASIC and analog-to-digital converter (ADC) chip, read the particle event information from the ASIC and ADC chip, and record the data in the event memory; 2) perform ASIC initialization and obtain event statistics and statistical results that are sent by the di-

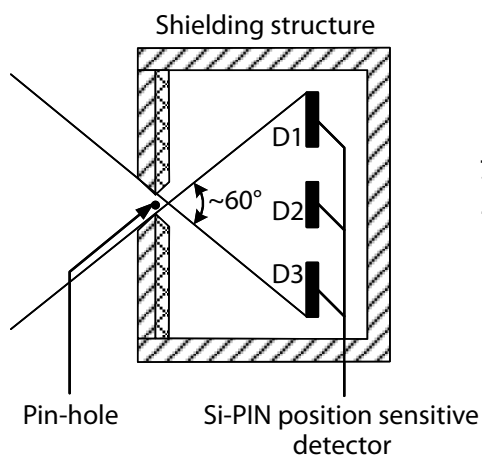

(a)

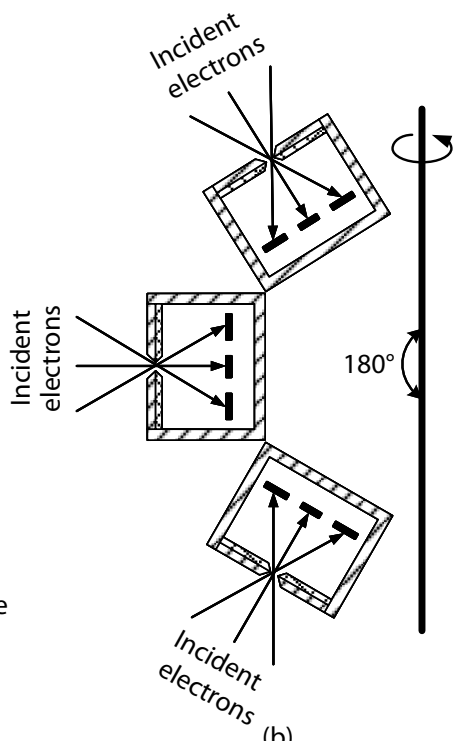

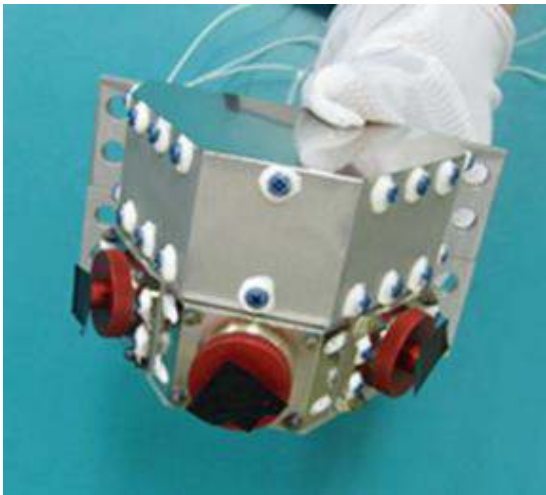

(c)

Figure 1. Diagram and photo of the MES sensor head. (a) A single detector cell with a $60^{\circ}$ angle detection range; (b) the structure consists of three sensors with a combined detection range of $\sim 180^{\circ}$; (c) Photo of the MES sensor head.

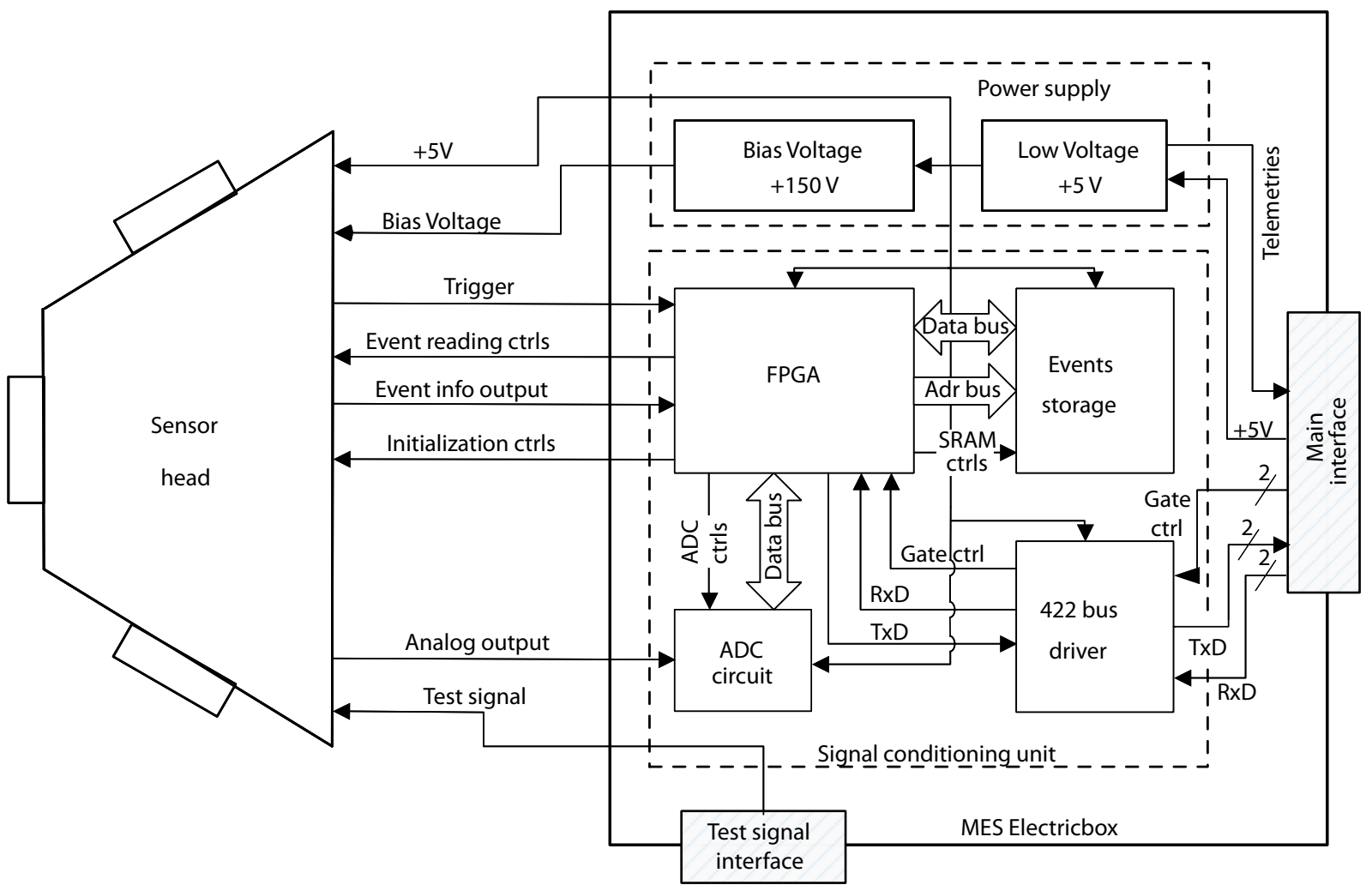

Figure 2. Block diagram of the MES. 
gital processor of the EEDP using RS-422 interface commands.

The SCU consists of the main control field-programmable gate array (FPGA) module, an ADC circuit module, an event storage module, and an RS-422 interface driver module. The ADC circuit module uses a 12-bit chip as the core; its main function is to quantify the voltage signal representing the particle energy in the particle event output by the ASIC. Due to the bipolar input feature of the ADC, 11 bits are available for energy encoding, and a sub-keV level quantification error can be achieved. The event storage module uses an SRAM chip (512KB) as the core, which stores the collected event information (including the energy code and direction code of each particle event). The FPGA is the main control module of the SCU. It receives the trigger signal from the sensor head, provides the corresponding event readout timing to determine the particle event information, and stores it in the event memory. It also receives and responds to commands from the 422-bus driver.

The main control FPGA of the SCU receives the gate control signal from the data processing unit (DPU) and sets the working status of the SCU accordingly. When the gate control signal is high, the SCU sets the event accumulation function to active, which means the FPGA responds to the event trigger signal from the sensor head and stores the events into local memory. When the gate control signal is low, the SCU enables the data access function. The FPGA receives serial commands from the digital processor and performs ASIC initialization, records the particle event statistics, and sends the statistical results to the DPU through the serial bus according to the command.

The low-voltage power supply ( $+5 \mathrm{~V}$ ) used by the MES is provided by the digital processor of the EEDP through the main connector, and the PWR module includes a DC-DC conversion circuit, which converts the low voltage into a bias signal $(+150 \mathrm{~V})$ and provides it to the sensor head during operation.

Figure 3 shows the flight model configuration of the MES. In contrast with the BD-IES (Zou H et al., 2018) on board the Chinese navigation satellite in IGSO, the MES has no dedicated DPU module and shares the DPU with the HED and DDCM. Therefore, the weight of the MES is reduced from $3.2 \mathrm{~kg}$ to $1.75 \mathrm{~kg}$. The perform-

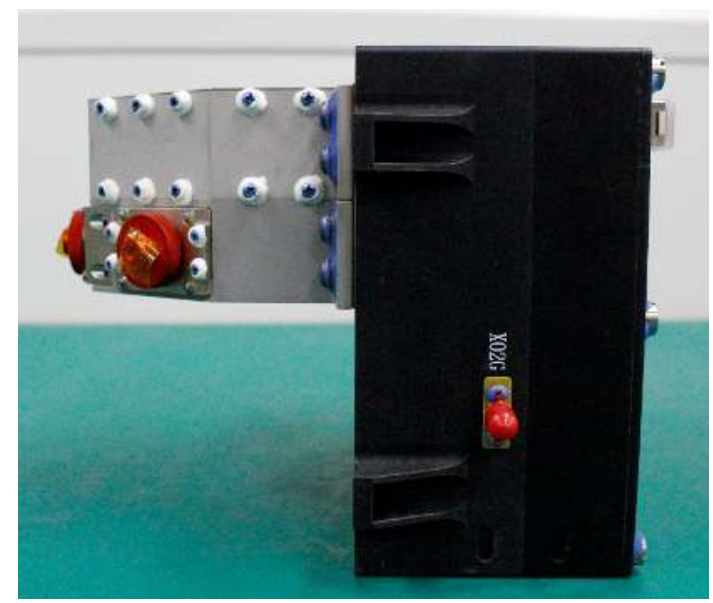

Figure 3. Photo of the flight-model MES. ance parameters of the MES are shown in Table 1.

\subsection{High-energy Electron Detector (HED)}

The HED is used to measure the flux of high-energy electrons from 0.5-3.0 MeV in the outer belt, incident in a fixed direction. VAP REPT utilizes an advanced and complex silicon telescope capable of precisely measuring the energy spectra of 1-20 MeV electrons (Baker et al., 2013a). In contrast, the HED sensor measurement energy range is not required to be very large $(0.5-3.0 \mathrm{MeV})$, utilizing a mature and straightforward $\Delta \mathrm{E}-\mathrm{E}$ silicon detector telescope.

Figure 4 shows the functional and structural diagram of the HED sensor head. A $50 \mu \mathrm{m}$ thick aluminum light-blocking layer is located in front of the telescope aperture to prevent photons and low-energy particles from entering the detectors. The front $\Delta \mathrm{E}$ detector uses a $100 \mu \mathrm{m}$ thick ion-implanted silicon PIN detector. The

Table 1. Characteristic parameters of the MES

\begin{tabular}{cc}
\hline Parameters & MES \\
\hline Particles & Electron \\
\hline Energy range $(\mathrm{keV})$ & $50-600$ \\
\hline & E1: $50-68$ \\
E2: $68-93$ \\
E3: $93-130$ \\
E4: $130-170$ \\
E5: $170-240$ \\
E6: $240-320$ \\
E7: $320-440$ \\
E8: $440-600$ \\
Underflow and overflow
\end{tabular}

\begin{tabular}{cc}
\hline Field-of-view & $30^{\circ} \times 180^{\circ}$ \\
\hline Angular coverage (range/intervals) & $180^{\circ} / 9$ \\
\hline Geometric factor $\left(\mathrm{cm}^{2} \cdot \mathrm{sr}\right)$ & $\sim 2.0 \times 10^{-3 *}$ (for each direction) \\
\hline
\end{tabular}

*The geometric factor is the average value of nine directions.

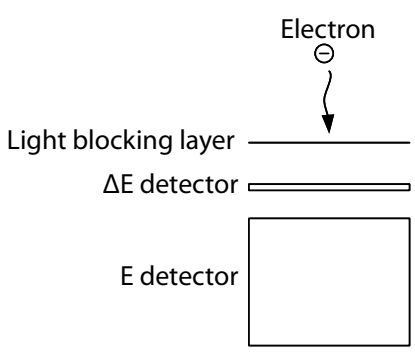

(a)

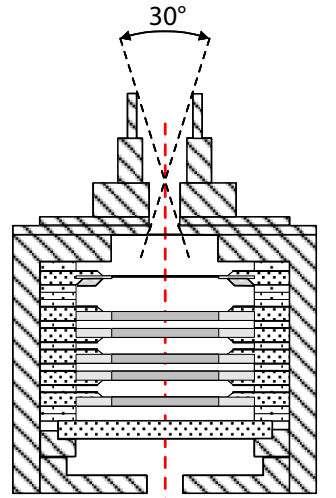

(b)
Figure 4. Diagram of the HED sensor head: (a) schematic diagram of the $\Delta \mathrm{E}$-E Si-detector telescope and (b) cross-section HED. 
back $E$ detector consists of five $1000 \mu \mathrm{m}$ ion-implanted silicon PIN detectors to form a $5000 \mu \mathrm{m}$ thick $\mathrm{E}$ detector, which can measure electron energies up to $3 \mathrm{MeV}$.

The smallest $L$-shell of the MEO satellite orbit is about 4.4 in the geomagnetic equator close to the center of the outer belt, where the high-energy electron flux is large. To avoid saturation of the HED's electronic system, the geometric factor of the HED sensor head is limited to $\sim 10^{-2} \mathrm{~cm}^{2} \cdot \mathrm{sr}$, therefore a copper collimator is used in front of the silicon detector telescope with an FOV of $\sim 30^{\circ}$. Figure $4 \mathrm{~b}$ shows a schematic diagram of the HED sensor head structure. The minimum thickness of the HED's sensor head housing is $5 \mathrm{~mm}$ of copper, preventing electrons $<7.5 \mathrm{MeV}$ or protons $<63 \mathrm{MeV}$ from reaching the silicon detectors through the HED housing walls. According to the energy spectra observed at the center of the outer belt in MEO (Fennell et al., 2000), electron flux with energies $>7.5 \mathrm{MeV}$ is more than three orders of magnitude less than for energies of $0.5-3.0 \mathrm{MeV}$ and can be ignored. The proton energy in the outer belt is usually less than $1 \mathrm{MeV}$ (proton pollution during solar proton events will be discussed separately); thus, proton contamination of the HED sensor head during periods without solar proton events can also be ignored.

Figure 5 shows the block diagram of the HED. The electronics box includes three units: the PA, SCU, and PWR. The main function of the $\mathrm{PA}$ is to amplify and shape the two signals from the $\Delta \mathrm{E}$ and $\mathrm{E}$ detectors, detect the peak $\mathrm{E}$ output, and perform digital conversion. The output signals of the two silicon detectors and the input signals are boosted and shaped by the amplifiers, proportional to the amplitude of the particle-deposited energy in the two detectors. The five silicon PIN detectors of the E detector are connected in parallel, and the signal is amplified and shaped by the $\mathrm{E}$ amplifier. This parallel connection results in a lower energy resolution, but the advantages are reduced weight and power consumption of the PA. The E amplifier output signal is divided into two channels: one is provided to the peak detection circuit, and the other is provided to an ADC. The peak detection circuit detects the peak of the $E$ amplifier output and informs the $A D C$ to start conversion, after which the FPGA reads the result. The output signal of the $\Delta \mathrm{E}$ detector amplifier is provided to the thre shold comparator, which compares the $\Delta \mathrm{E}$ amplifier's output signal with the preset threshold to distinguish between incident electrons and ions. Typically, the $\Delta \mathrm{E}$ detector signal generated by electrons is much smaller than that of ions, and can thus be excluded when the amplitude of the $\Delta \mathrm{E}$ detector output signal exceeds a threshold level determined by calibration. The threshold comparator then outputs a veto signal to the FPGA, which completes the processing of the energy code of the particle event accordingly.

In addition to the main control FPGA, the SCU of the HED also contains an event memory and an RS-422 driver. Once the main control FPGA has obtained the energy code of the particle event from the E output, it determines whether the event is valid by coincidence with the $\Delta \mathrm{E}$ output. If the event is indeed valid, the FPGA places the event information into the event memory, which is an SRAM with $128 \mathrm{~KB}$ capacity. Similar to the MES, the SCU of the HED also uses a gate control signal to determines the working status of the FPGA.

The low-voltage power supply used by the HED is provided separately by the EEDP digital processor through the main connector. The PWR module includes a DC-DC conversion circuit, which generates two bias voltages of $-20 \mathrm{~V}$ and $-170 \mathrm{~V}$ for the silicon PIN detectors in the HED sensor head.

Figure 6 shows a photo of the flight-model HED. The sensor head of the HED is partially embedded in the electronic box to increase the shielding thickness of the sensor head laterally. The performance parameters of the HED are listed in Table 2.

\subsection{Deep Dielectric Charging Monitor (DDCM) and Digital Processor}

The DDCM of the EEDP uses an FR4 circuit board as the base material, which is commonly used in aerospace payloads. A function-

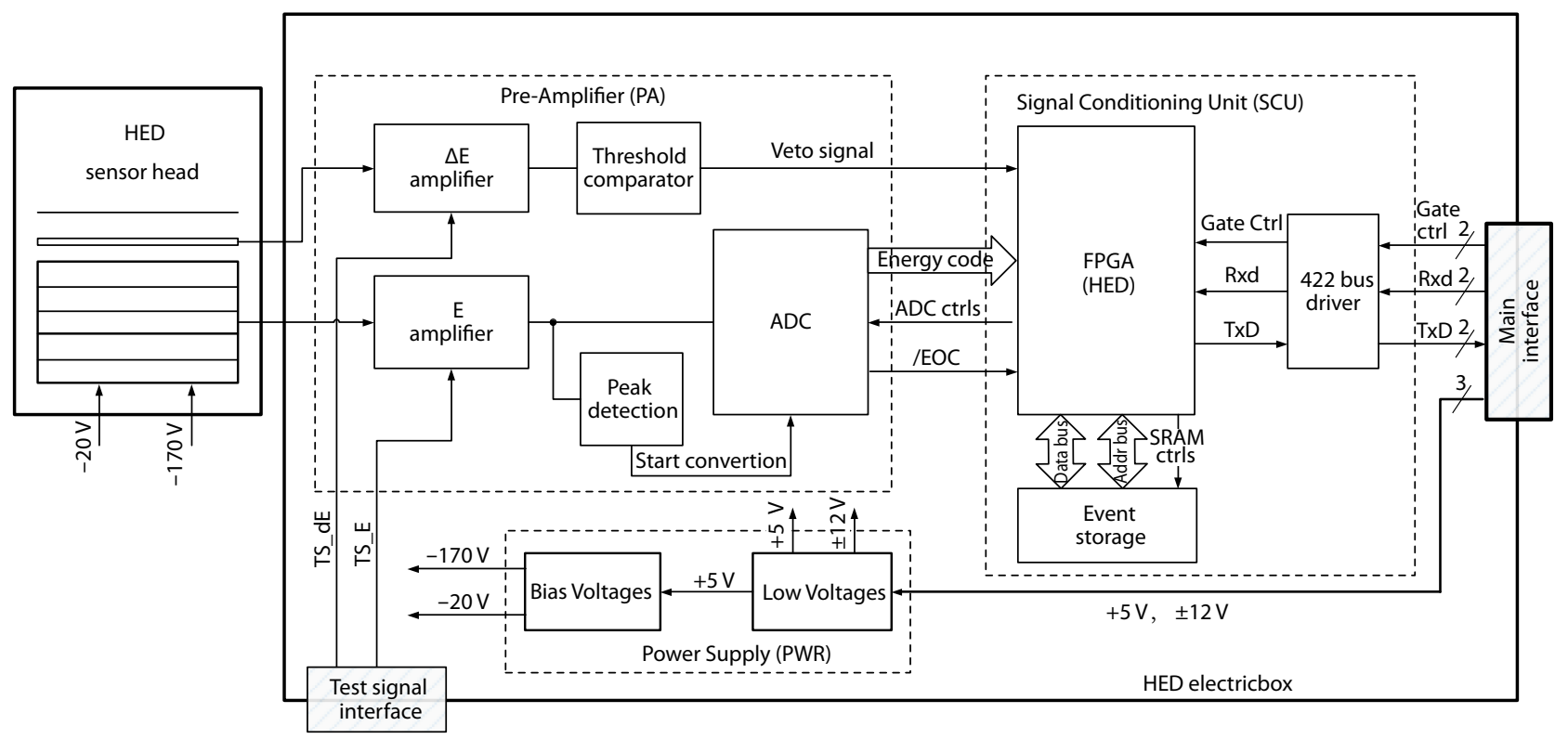

Figure 5. Block diagram of the HED. 


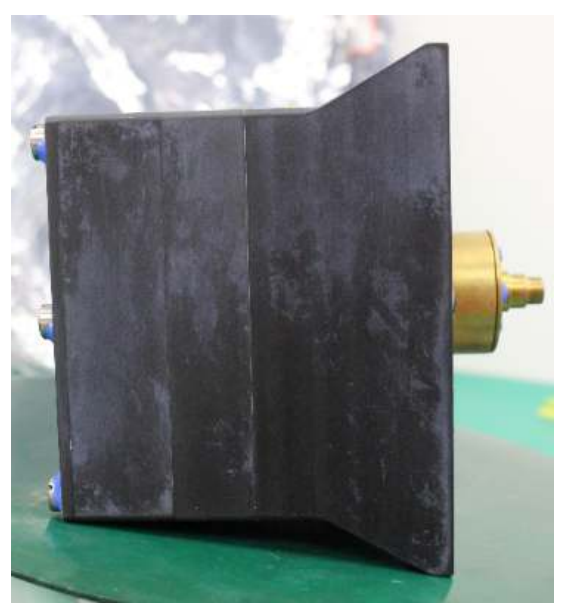

Figure 6. Photo of the flight-model HED.

al diagram of the DDCM sensor head is shown in Figure 7. Copper layers at the top and bottom of the DDCM sensor head measure the deep dielectric charging current, while the middle copper plates are sampling electrodes that measure the charging potentials. The sensor material is FR4 with an area of $\sim 82.3 \mathrm{~cm}^{2}$ and thickness of $1.6 \mathrm{~mm}$. When the charged particle flow enters the DDCM sensor, a charging current is formed on the upper and lower layers. When the number of incident charged particles exceeds the charges leaked from the middle metal layers, a charging voltage accumulates on the middle layers.

Figure 8 shows the functional block diagram of the DDCM. The
Table 2. Characteristic parameters of HED.

\begin{tabular}{cc}
\hline Parameters & HED \\
\hline Particles & Electron \\
\hline Energy range $(\mathrm{MeV})$ & $0.5-3.0$ \\
\hline & E1: $0.5-0.8$ \\
Energy channel $(\mathrm{MeV})$ & E2: $0.8-1.2$ \\
& E3: $1.2-2.0$ \\
& E4: $2.0-3.0$ \\
\hline Field-of-view & Underflow and overflow \\
\hline Geometric factor $\left(\mathrm{cm}^{2} \cdot \mathrm{sr}\right)$ & $30^{\circ}$ cone-angle \\
\hline
\end{tabular}

DDCM consists of a sensor head, a PA, a main amplifier, and a filter circuit. The outputs of the DDCM are four amplified analog voltage signals, which are proportional to the charging currents and the charging potentials generated by the sensor head. After passing through the sampling circuits, the outputs of the DDCM sensor head are transmitted to the PA and the main amplifier and are low-pass filtered to remove high-frequency noise. The output signals of the DDCM are four amplified quasi-DC voltage signals, which are provided to the DPU of the digital processor for storage.

The DDCM is housed in the same case as the digital processor, which is also shared with the MES and HED. The functional diagram of the digital processor is shown in Figure 9. The digital processor includes a DPU and a PWR. The DPU includes an FPGA, an

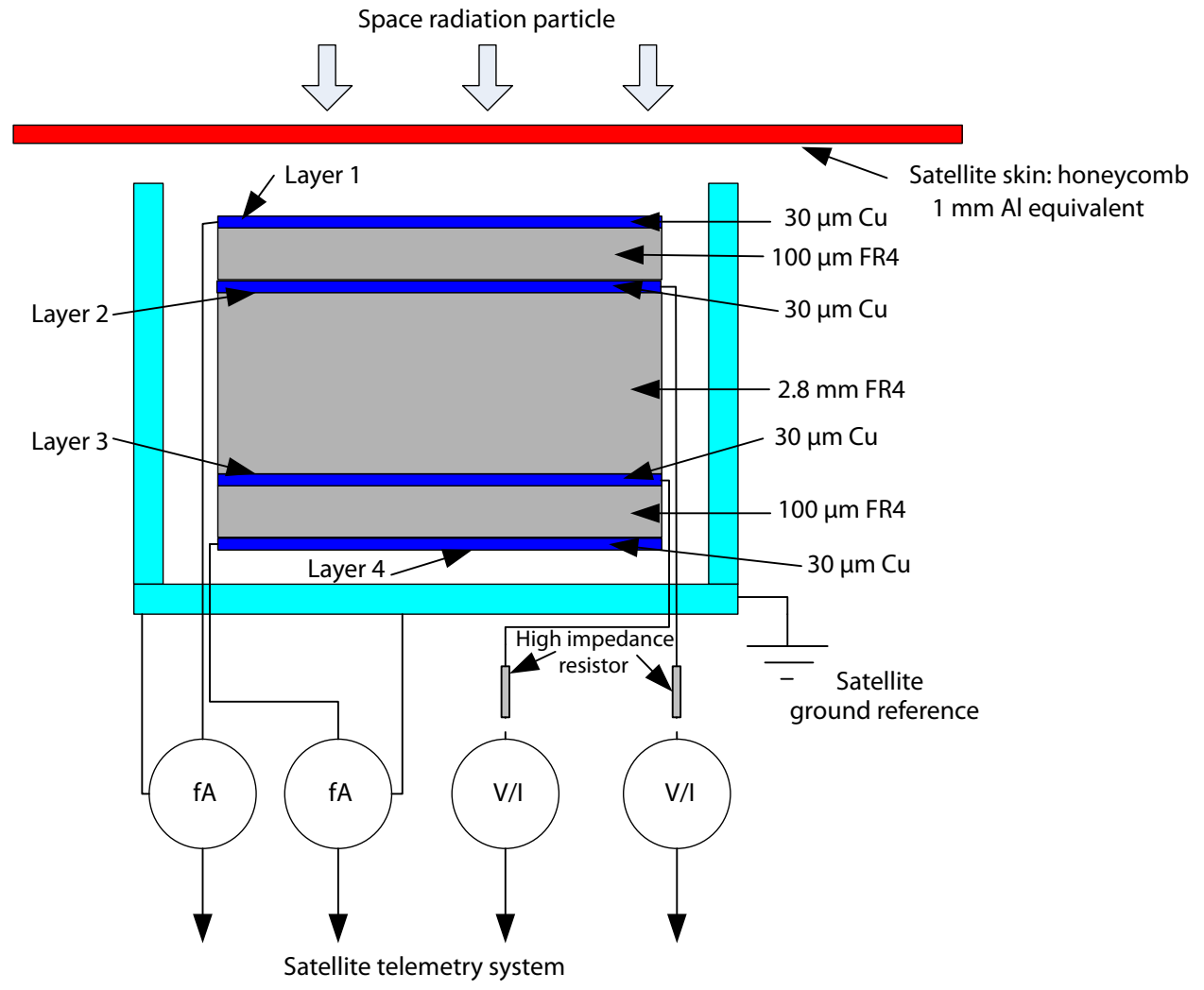

Figure 7. Diagram of the sensor head of the DDCM. 


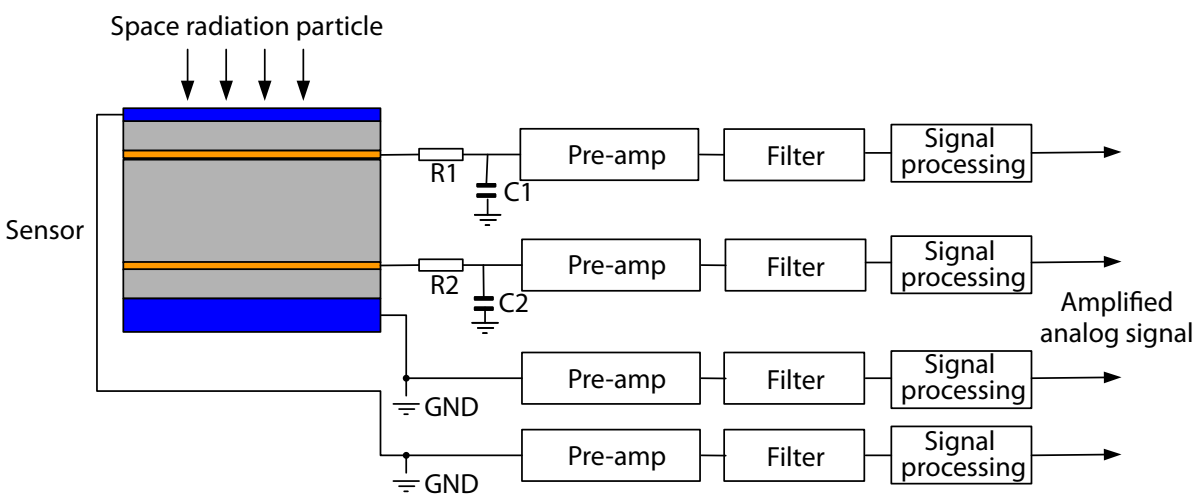

Figure 8. Functional block diagram of the DDCM.

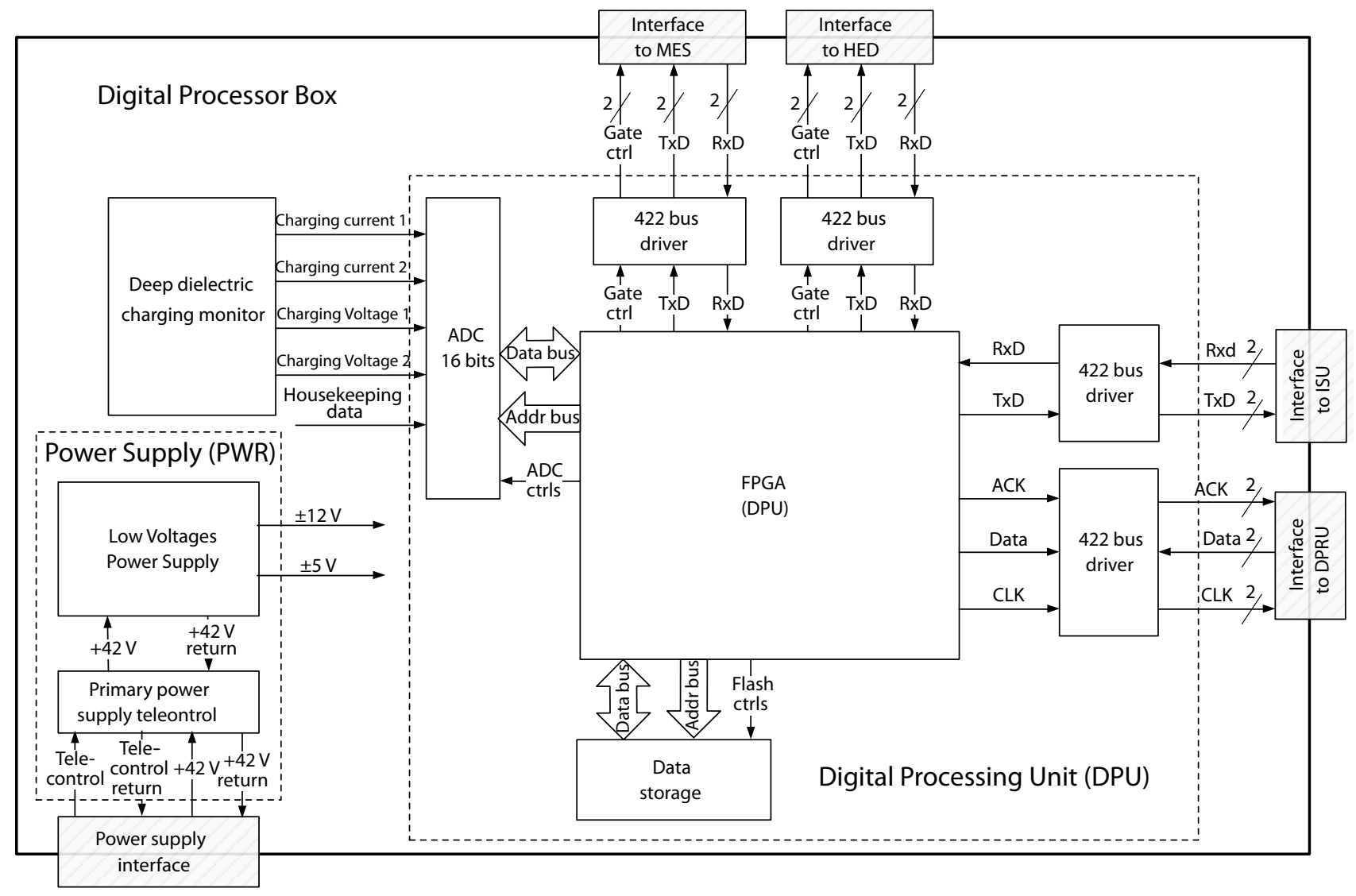

Figure 9. Block diagram of the Digital Processor.

ADC (16 bit), 4 groups of RS-422 drivers, and data memory. The FPGA receives the commands from the integrated service unit (ISU) through the serial bus and completes the command response. The FPGA sends scientific data to the data processing and routing unit (DPRU) through the synchronous serial bus. The FPGA also sends data acquisition and initialization-related commands to the MES and HED through serial buses and receives scientific data from them. The FPGA uses the RS-422 driver to provide sampling gate control signals to the MES and HED which collect and store particle events when the gate control signal is high, allowing control of the sampling gate opening time. According to the task schedule, the FPGA provides data acquisition timing to the 16-bit ADC to sample the four outputs of the DDCM and the housekeeping data, which are timestamped and combined with MES and HED data and sent to the DPRU. Thus these tasks dictate the observation time resolution of medium-energy electrons, high-energy electrons, deep charging currents, and voltages at a $1 \mathrm{~Hz}$ sampling rate.

The PWR module of the digital processor receives the on/off switch from the satellite and provides a low voltage supply for the whole instrument package.

The main functions of the digital processor are as follows:

$>$ Perform the initialization of the digital processor;

$>$ Perform commands, receive and respond to communications with the ISU; 
$>$ Collect housekeeping information from the instruments;

> Communicate with the MES, to complete initialization and scientific data acquisition; provide the gate control signal to the MES and control the sampling gate opening time (200 ms);

$>$ Communicate with the HED to complete scientific data acquisition; provide the gate control signal to the HED and control the sampling gate opening time (300 ms);

$>$ Acquire DDCM output signals;

$>$ Store the scientific data package from each sensor;

$>$ Communicate with and send scientific data packets to the DPRU.

Figure 10 shows photos of the DDCM and the digital processor. Before launching the payload, the protective layer of the DDCM is removed to expose the sensor and allow high-energy electrons to enter. The performance parameters of the DDCM are listed in Table 3.

\subsection{Instrument Mounting Position and Satellite Orbit}

The EEDP is installed on three Chinese navigation satellites in MEO, stabilized in three axes. The instrument is installed on the back of the satellite to prevent sunlight from interfering with the instrument. The electronics boxes of the MES and HED are located inside the satellite, and the latter's sensor head extends outside of the chassis to ensure that the FOV is not blocked. Since the satellite is a three-axis stabilized platform and is in a constant-yaw state, the detection direction of the MES and HED changes with the satellite's movement. Since the satellite does not measure the local magnetic field, the pitch angles corresponding to the directions of the MES and HED sensor heads cannot be determined directly. However, due to the mounting direction of the MES and HED as the satellite moves close to the Earth's equatorial plane, the central direction of the MES sensor head is nearly perpendicular to the local magnetic field, as shown in Figure 11.

The EEDPs were installed on three Chinese navigation satellites: MEO1, MEO2, and MEO3, at an orbital altitude of $21500 \mathrm{~km}$, an inclination of $55^{\circ}$, and an orbital period of 12.88 hours. MEO1 and MEO2 were launched at the end of October 2018, and MEO3 was launched at the end of November 2019. Figure 12 shows the typical orbits of the three MEO satellites on January 1, 2020. MEO1 and MEO2 are in the same orbital plane, but the phase difference
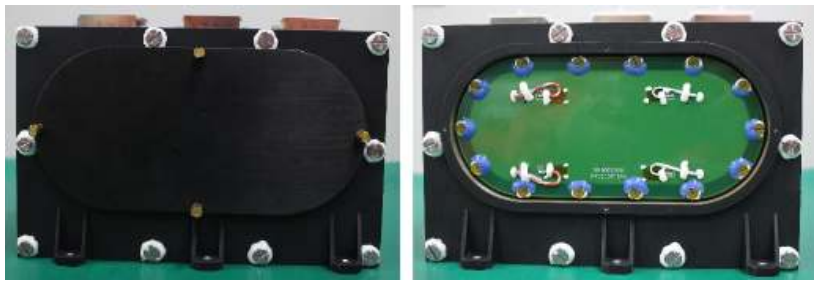

Figure 10. Photos of the DDCM and the digital processor.

Table 3. Characteristic parameters of the DDCM

\begin{tabular}{cc}
\hline Parameters & DDCM \\
\hline Charging Voltage & $-2.5 \mathrm{kV}$ to 0 V \\
Charging Current & $0.01-50 \mathrm{pA}$ \\
\hline
\end{tabular}

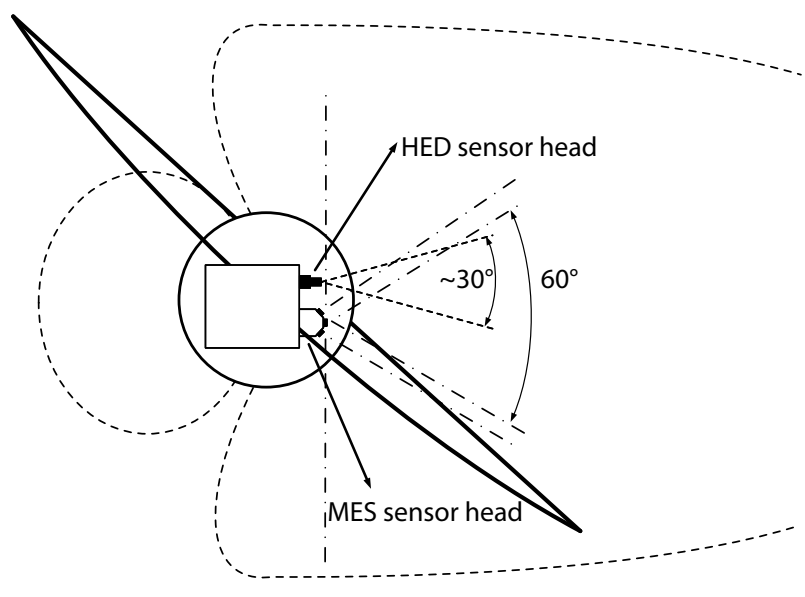

Figure 11. Diagram of the pointing directions of the MES and HED sensor heads in orbit. The solid line is the MEO of the satellite, the dashed lines show the geomagnetic field, the dash-dot lines show the field angle ranges of the MES sensor head units, and the dotted lines show the field angle ranges of the HED sensor head. The circle shows the position of the Earth while the square represents the satellite platform.

is $90^{\circ}$. MEO3 is on another orbital plane.

The MEO satellites cross the outer belt twice in each orbit, so their coordinated measurements can improve the sampling time resolution of the energetic electrons in the outer belt. Figure 13 shows the change in $L$-shell over time for the three MEO satellites on January 1, 2020. Spanning $L=4.4-18$, this compensates for VAP's insufficient coverage of the larger $L$-shells. Additionally, the combination of the three satellites' instruments reduces the sampling time interval for the outer radiation zone center $(L \sim 4-5)$ to $\sim 3.3$ hours, while also increasing the temporal resolution of energetic electron detection in the outer belt.

\section{Instrument Testing and Calibration}

\subsection{MES Testing and Calibration}

The sensor head of the MES is the same as that of the BD-IES; thus, testing and calibration are identical. For details, please refer to Zou $\mathrm{H}$ et al. (2018). A radioactive source $\left({ }^{207} \mathrm{Bi}\right)$ and an analog signal source were used to perform energy calibration and system performance tests on the BD-IES sensor head. Figure 14 shows the energy spectra for the ${ }^{207} \mathrm{Bi}$ source measured by the three directional channels of the MES sensor head unit 1 on board MEO3.

The silicon detectors of the unit 1 sensor head measured the typical peaks in the spectrum of ${ }^{207} \mathrm{Bi}: 74.2 \mathrm{keV}, 481.6 \mathrm{keV}, 554.0 \mathrm{keV}$, $975.6 \mathrm{keV}$, and $1048.1 \mathrm{keV}$. The peak at $74.2 \mathrm{keV}$ is the most prominent, whereas the peak at $975.6 \mathrm{keV}$ is relatively low. These energy spectrum characteristics are consistent with the energy response of the BD-IES using ${ }^{207} \mathrm{Bi}$ (Zou H et al., 2018).

The number of central ADC channels corresponding to the spectrum peaks shown in Figure 14 can be obtained by Gaussian function fitting. Table 4 lists the number of central ADC channels corresponding to the four typical peaks of the ${ }^{207} \mathrm{Bi}$ radioactive source measured by the nine directional channels of the MES 

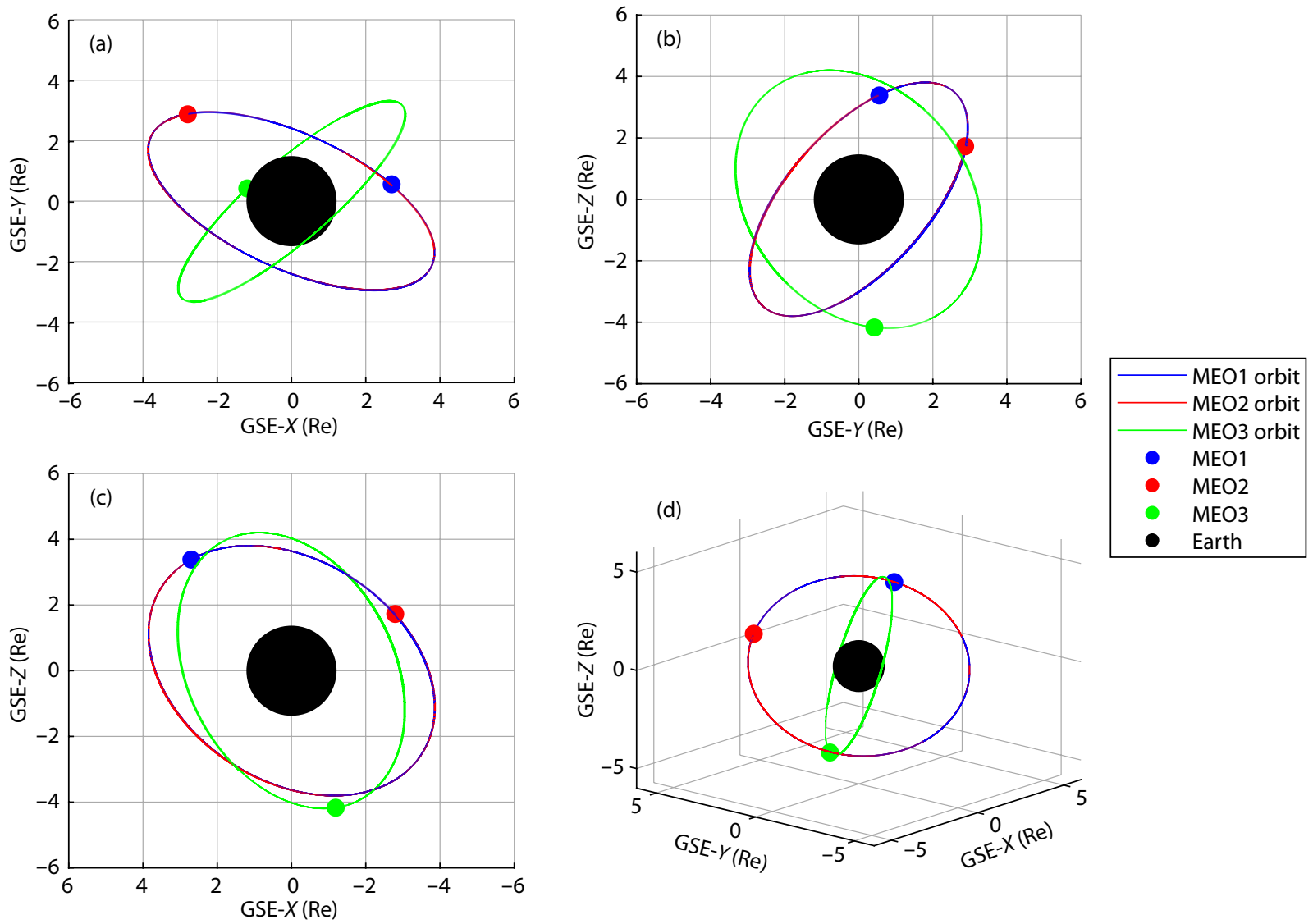

Figure 12. The typical orbits of the three MEO navigation satellites in geocentric solar ecliptic (GSE) coordinates on Jan 1, 2020.

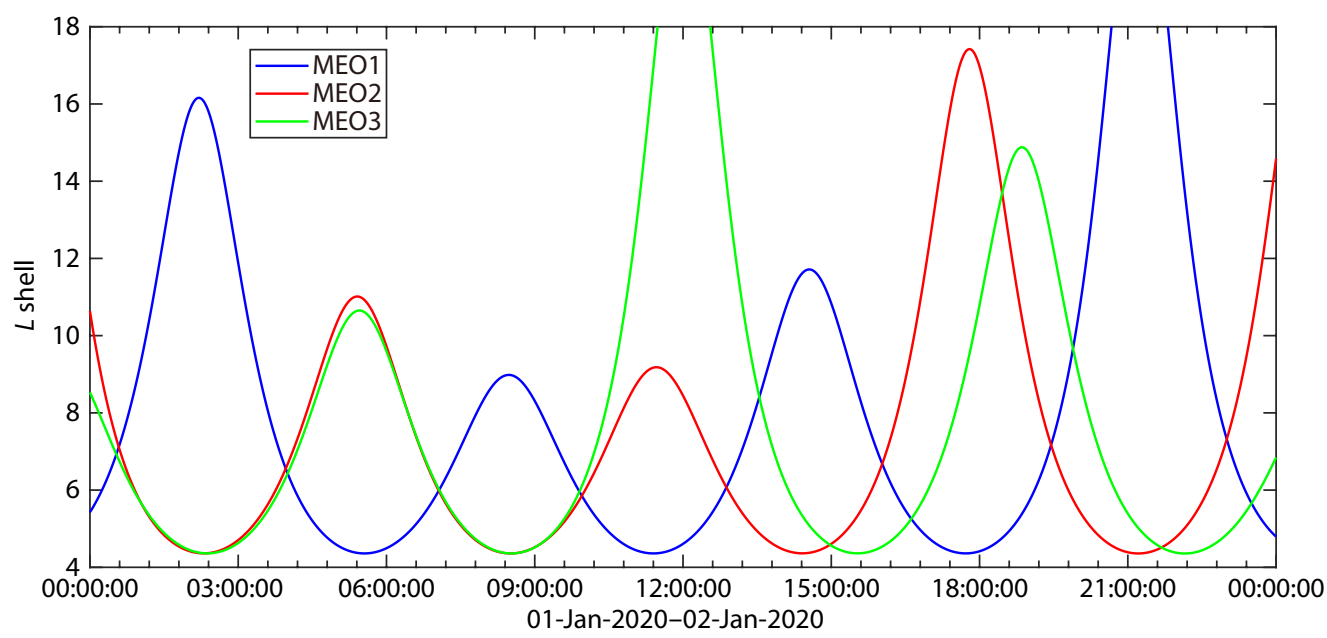

Figure 13. The temporal variations of the three MEO satellites' orbits in L-shell on Jan 12020.

sensor head.

A ${ }^{133} \mathrm{Ba}$ radioactive source was also used to measure the energy response of the MES, showing two peaks with a relatively large flux at $31 \mathrm{keV}$ and $81 \mathrm{keV}$. In addition, in the higher energy range, ${ }^{133} \mathrm{Ba}$ has several peaks with smaller fluxes: $276 \mathrm{keV}, 303 \mathrm{keV}, 356$ $\mathrm{keV}$, and $384 \mathrm{keV}$.

Figure 15 shows the energy spectra for ${ }^{133} \mathrm{Ba}$ in the low- and highenergy ranges measured with channel 1 (corresponding to ASIC channel 5) of the MES sensor head, showing the six characteristic peaks of this source.

The central ADC channels corresponding to the peaks in the energy spectra shown in Figure 15 can be obtained by using Gaussian function fitting. Table 5 lists the central ADC channels corresponding to the six typical peaks for ${ }^{133} \mathrm{Ba}$ measured by the nine directional channels of the MES sensor head.

The linearity of the energy response in each directional channel of the MES sensor head can be derived from the relationship between the central ADC channels and the energies of the typical 
Table 4. The central ADC channel numbers $(C C N)$ of the four typical peaks of the ${ }^{207} \mathrm{Bi}$ source measured by the nine directional channels of the MES sensor head.

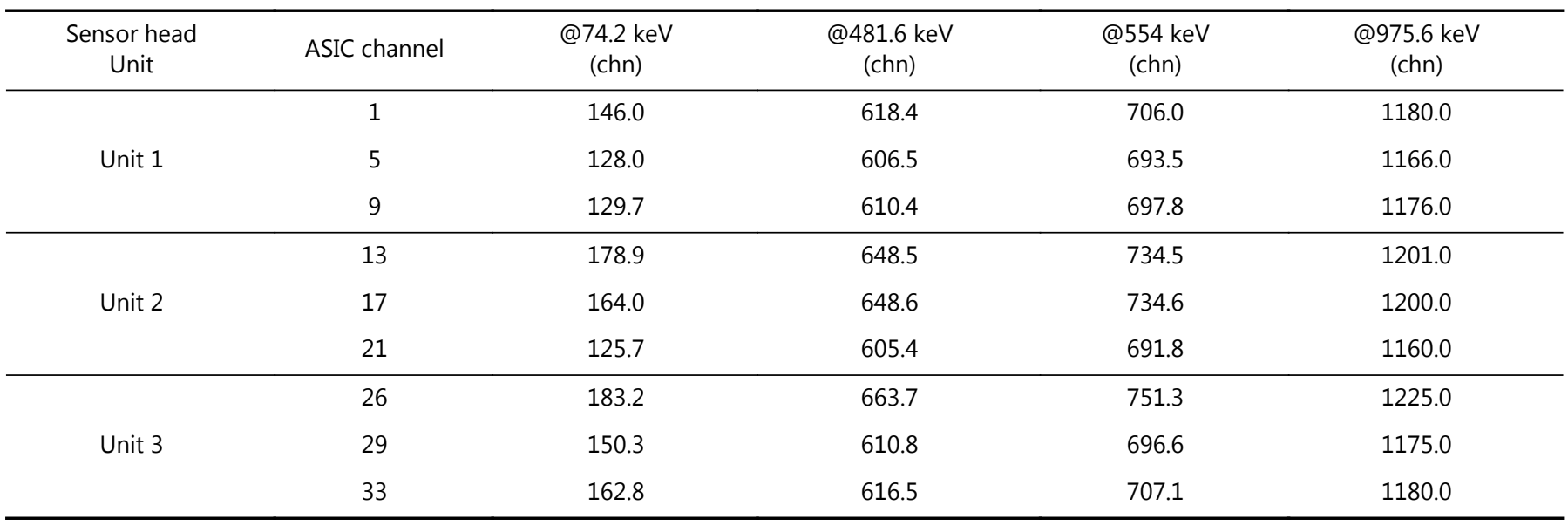

Table 5. The central ADC channels of the six typical peaks of ${ }^{133}$ Ba source measured by nine directional channels of the MES sensor head.

\begin{tabular}{cccccccc}
\hline $\begin{array}{c}\text { Sensor head } \\
\text { Unit }\end{array}$ & ASIC channel & $\begin{array}{c}\text { @31 keV } \\
\text { (chn) }\end{array}$ & $\begin{array}{c}\text { @81 keV } \\
\text { (chn) }\end{array}$ & $\begin{array}{c}\text { @276 keV } \\
\text { (chn) }\end{array}$ & $\begin{array}{c}\text { @303 keV } \\
\text { (chn) }\end{array}$ & $\begin{array}{c}\text { @356 keV } \\
\text { (chn) }\end{array}$ & $\begin{array}{c}\text { @384 keV } \\
\text { (chn) }\end{array}$ \\
\hline & 1 & 94.6 & 153.8 & 382.3 & 414.2 & 478.7 & 511.6 \\
Unit 1 & 5 & 74.3 & 136.3 & 374.1 & 406.3 & 463.4 & 496.1 \\
& 9 & 78.3 & 139.0 & 372.2 & 404.5 & 468.6 & 501.9 \\
\hline Unit 2 & 13 & 130.8 & 186.4 & 413.6 & 444.7 & 540.5 & 53.6 \\
& 17 & 111.9 & 173.1 & 407.4 & 439.8 & 504.6 & 463.2 \\
\hline & 21 & 75.2 & 134.7 & 367.4 & 398.8 & 496.5 \\
\hline
\end{tabular}
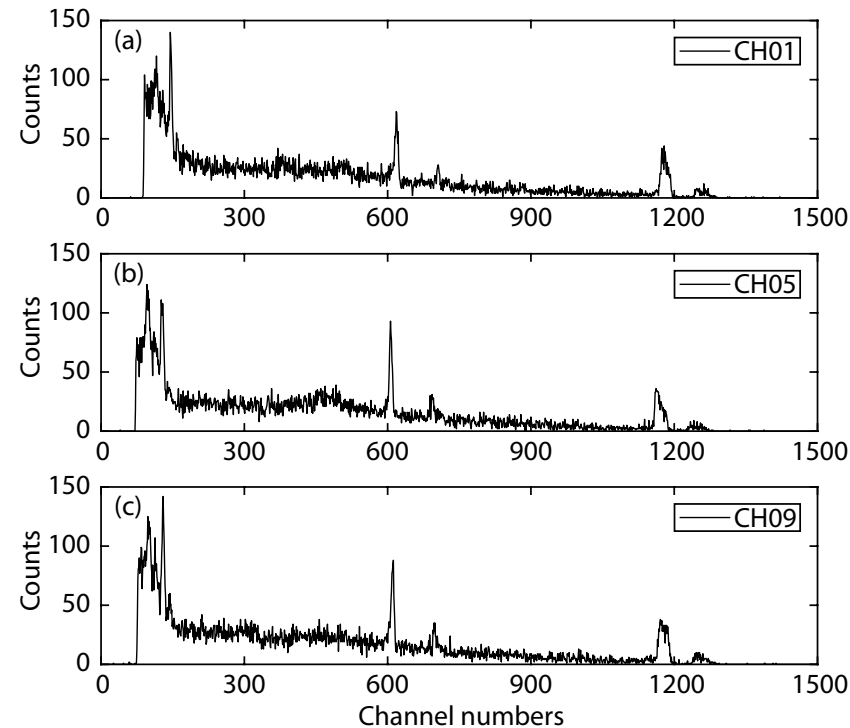

Figure 14. Energy spectra of ${ }^{207} \mathrm{Bi}$ measured by the silicon detector 1 (a), 2 (b), and 3 (c) in unit 1 of the MES sensor head. The channels 1, 5, and 9 are the ASIC channels connecting the three detectors.

spectral peaks for the ${ }^{207} \mathrm{Bi}$ and ${ }^{133} \mathrm{Ba}$ radioactive sources. Figure 16 shows the relationship between the central ADC chan-
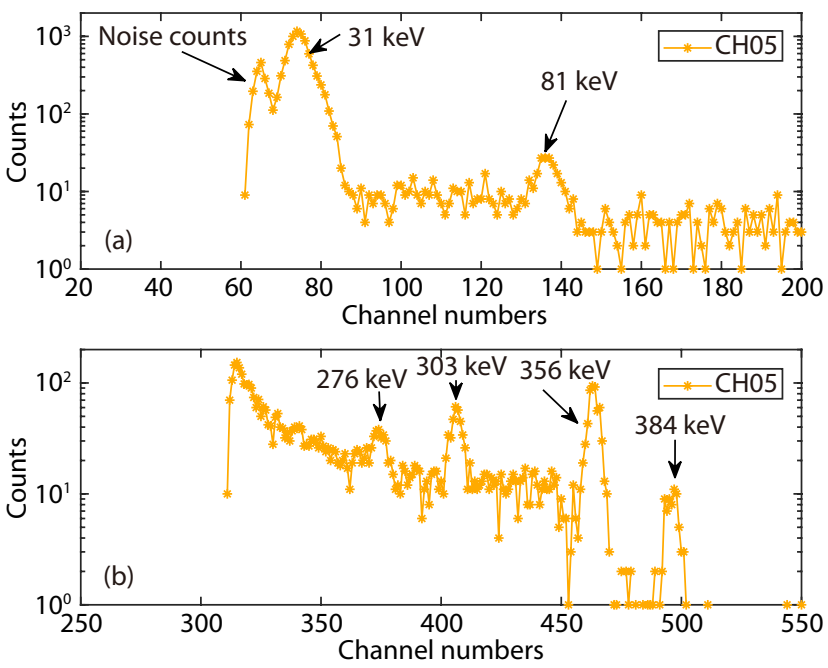

Figure 15. Energy spectra of ${ }^{133} \mathrm{Ba}$ measured by directional channel 1 of the MES sensor head for the (a) low-energy spectrum, and (b) high-energy spectrum.

nels corresponding to 10 peaks measured by the nine directional channels of the MES sensor head, with four typical peaks of ${ }^{207} \mathrm{Bi}$ and six typical peaks of ${ }^{133} \mathrm{Ba}$; the black lines are the linear fitting 

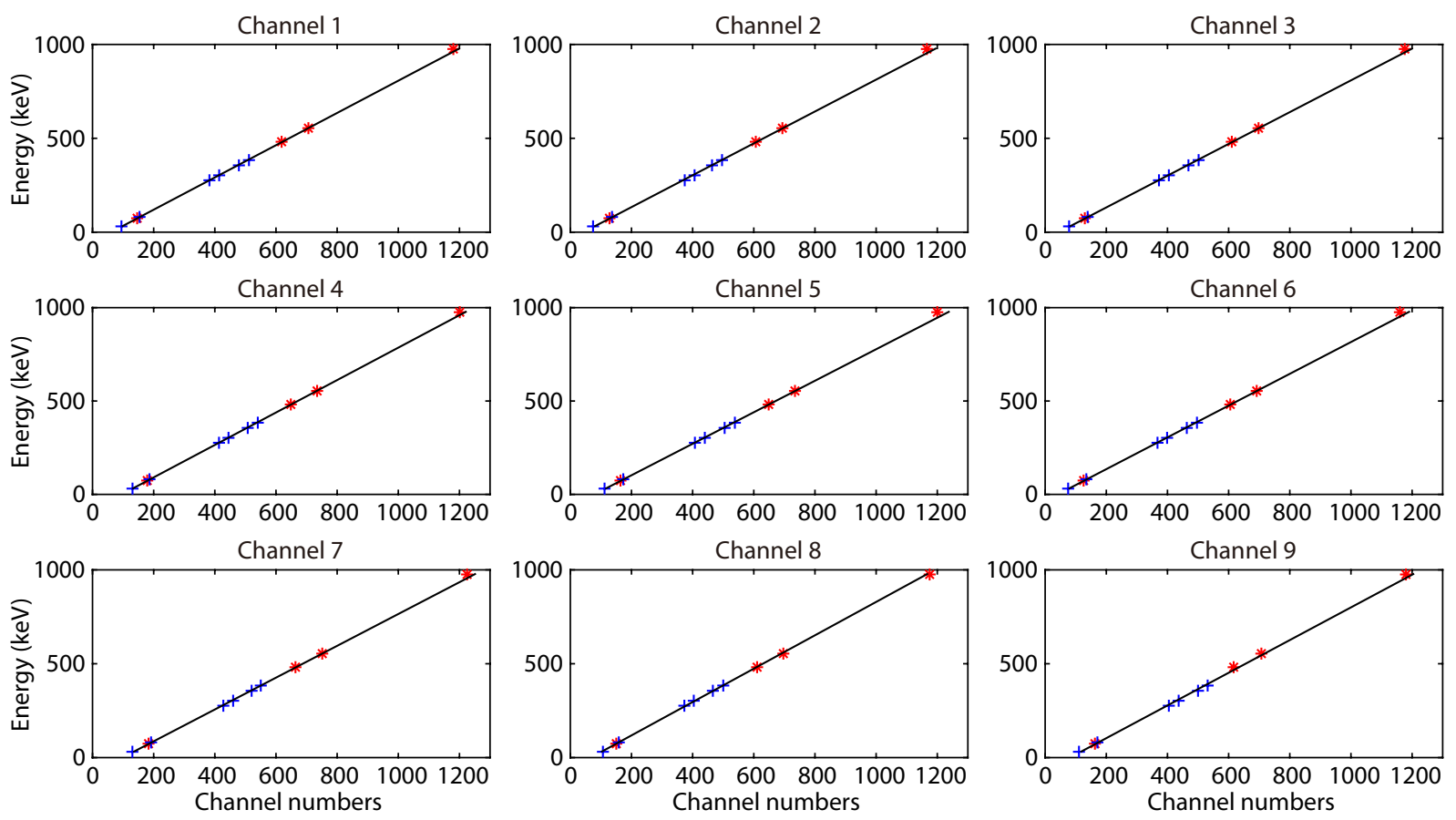

Figure 16. The energy linearity of nine directional channels of the MES sensor head, with black lines showing the fitting results. The abscissa is the ADC channel number, and the ordinate is the peak energy in keV. The red ' $*$ ' corresponds to the central channels of the four ${ }^{207} \mathrm{Bi}$ energy peaks at $74 \mathrm{keV}, 481.6 \mathrm{keV}, 554 \mathrm{keV}$, and $976.4 \mathrm{keV}$. The blue ' + ' corresponds to the central channels of the six peaks at $31 \mathrm{keV}, 81 \mathrm{keV}, 276 \mathrm{keV}, 303 \mathrm{keV}, 356 \mathrm{keV}$, and $384 \mathrm{keV}$.

results, confirming that the energy responses of the nine directional channels of the MES sensor head exhibit good linearity in the energy range of 50-600 keV.

The linear fitting result of the measured central ADC channel and peak energy can be described using the following equation:

$$
E=\mathrm{EperCh} \times(\mathrm{Ch}-\mathrm{Ch} 0),
$$

where $E$ is the electron energy collected by the detector; EperCh is the channel width or energy per channel; and $\mathrm{ChO}$ is the starting channel number, i.e., the channel number for $0 \mathrm{keV}$ electron input. Table 6 lists the channel width (energy per channel) and the starting channel numbers of the nine directional channels of the MES sensor head obtained by linear fitting. The linearity of each directional channel is calculated from the linear fitting results. The maximum deviation is about $1.6 \%$ and the average is about $0.4 \%$. According to Equation (1) and Table 6, we can calculate the ADC channel numbers corresponding to the nine energy boundary thresholds of the eight energy bands for each directional channel. These ADC channel numbers are the energy boundary thresholds sent from the DPU to the MES to obtain the counts of the eight energy bands in each direction.

A test signal source was used to measure the system electronics noise of each MES directional channel and verify its performance, using the same method used to test the BD-IES (Zou $\mathrm{H}$ et al., 2018). The MES directional channel response to a fixed-amplitude test signal is the Gaussian-distributed count in a series of ADC channels. The full width at half maximum (FWHM) of the Gaussian-distributed counts reflects the electronic system's noise for each of the nine directional channels, shown in Table 7. The MES minimum, maximum, and average system electronics noise for the nine channels was $2.63 \mathrm{keV}, 5.92 \mathrm{keV}$, and $4.94 \mathrm{keV}$, respectively. Therefore, the system electronics noise level of the MES is better than that of the BD-IES.

The angular resolution of the pinhole imaging system was verified using the observations of Polar-IES and Cluster-IES. For the direction calibration test of the BD-IES sensor head, we used a Monte Carlo simulation and a collimated electronic radiation source $\left({ }^{90} \mathrm{Sr} /{ }^{90} \mathrm{Y}\right.$ ) to test the directional response ( $\mathrm{Zou} \mathrm{H}$ et al., 2018), shown in Figure 17. For detailed test methods and procedures, please refer to Zou H et al. (2013).

Figure 17 shows that the FWHM directional response curves of the three directions of the BD-IES sensor head unit are all less than $20^{\circ}$, indicating good angular resolution of the pinhole imaging structure. Since the design of the MES imaging sensor head is identical to BD-IES, it has the same angular resolution.

Geant4 was used to perform a Monte Carlo simulation of the geometric factors for each direction and energy band of the MES sensor head. The results are consistent with those shown in Table 1 using the method described by Zou H et al. (2019). Geant4 is also used to simulate the anti-proton contamination performance of the MES sensor head, which is acceptably close to that of the BD-IES (Luo L et al., 2015; Zou H et al., 2019).

\subsection{HED Testing and Calibration}

A ${ }^{207} \mathrm{Bi}$ radioactive source and an analog test signal source were used for the energy calibration and system performance test of the HED sensor head. By adjusting the FPGA software in the HED's 
Table 6. The channel widths and the starting channel numbers of the nine directional channels of the MES sensor head.

\begin{tabular}{cccc}
\hline $\begin{array}{c}\text { Sensor head } \\
\text { Unit }\end{array}$ & $\begin{array}{c}\text { ASIC } \\
\text { channel }\end{array}$ & $\begin{array}{c}\text { Channel width } \\
\text { keV/ch }\end{array}$ & $\begin{array}{c}\text { Origination channel } \\
\text { Ch0 }\end{array}$ \\
\hline \multirow{2}{*}{ Unit 1 } & 1 & 0.8589 & 60.6 \\
& 5 & 0.8489 & 42.7 \\
Unit 2 & 9 & 0.8461 & 44.3 \\
\hline \multirow{3}{*}{ Unit 3 } & 13 & 0.8686 & 95.2 \\
& 21 & 0.8435 & 78.6 \\
& 26 & 0.8508 & 41.1 \\
\hline & 33 & 0.8476 & 97.6 \\
& 29 & 0.8886 & 66.8 \\
\hline
\end{tabular}

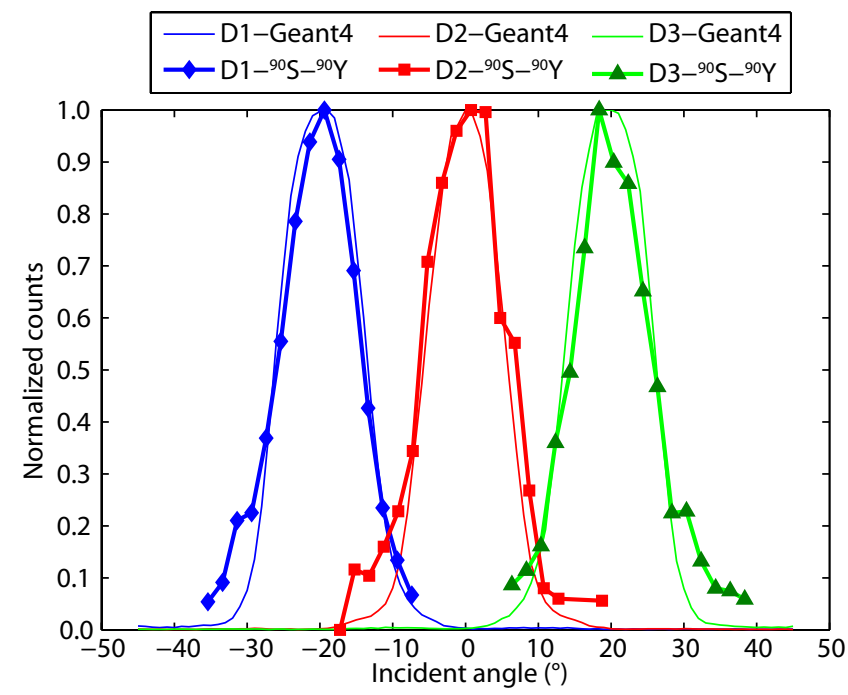

Figure 17. The normalized angular response curves for the three detectors in the BD-IES sensor head Unit 2 (D1 (blue line), D2 (red line), and D3 (green line)) obtained from the collimated $\beta$ source test (bold lines) and the Geant4 simulation test with a cone electron source (thin lines) (Zou $\mathrm{H}$ et al., 2013).

SCU, it can operate in multi-channel mode (2048 channels) to analyze the energy response to the incident electrons. Figure 18 shows the energy spectrum for ${ }^{207} \mathrm{Bi}$ measured by the HED sensor head on MEO1. The two peaks (976.4 keV and 1048.1 keV) on the right side of the energy spectrum are the characteristic peaks of ${ }^{207} \mathrm{Bi}$, while another characteristic peak $(481.6 \mathrm{keV})$ is at the left side of the energy spectrum. The HED sensor head could not measure the $74 \mathrm{keV}$ characteristic peak of ${ }^{207} \mathrm{Bi}$ source, however, primarily due to an increase in the equivalent capacitance of the $E$ detector caused by the parallel connection of the five silicon PIN detectors. Additionally, there is significant system electronic noise of the amplifier circuit since it is composed of traditional discrete components.

The energy spectrum shown in Figure 18 is the multi-channel analysis result of the $E$ detector output signal. The energy of emitted source electrons in the light barrier and $\Delta \mathrm{E}$ detector is not part of
Table 7. The FWHM in the ADC channels of test signal spectra measured by the nine directional channels of the MES, and the system electronics noise (at room temperature, $25^{\circ} \mathrm{C}$ ).

\begin{tabular}{cccc}
\hline $\begin{array}{c}\text { Sensor head } \\
\text { Unit }\end{array}$ & $\begin{array}{c}\text { ASIC } \\
\text { channel }\end{array}$ & $\begin{array}{c}\text { FWHM } \\
\text { (ADC channels) }\end{array}$ & $\begin{array}{c}\text { System electronics } \\
\text { noise (keV) }\end{array}$ \\
\hline \multirow{3}{*}{ Unit 1 } & 1 & 5.07 & 4.35 \\
& 5 & 5.57 & 4.73 \\
Unit 2 & 9 & 5.72 & 4.84 \\
\hline \multirow{3}{*}{ Unit 3 } & 13 & 6.82 & 5.92 \\
& 21 & 6.14 & 5.18 \\
& 26 & 6.35 & 5.40 \\
\hline & 33 & 6.10 & 2.63 \\
& 29 & 6.50 & 5.75 \\
\hline
\end{tabular}

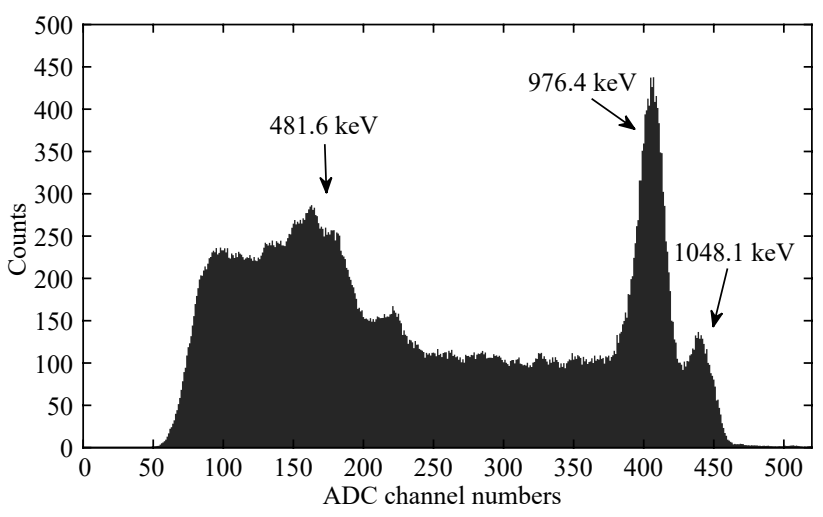

Figure 18. Energy spectra of ${ }^{207} \mathrm{Bi}$ measured by the HED sensor head.

this analysis. Therefore, when investigating the linear relationship between the central ADC channels of the characteristic peaks measured by the HED and the characteristic peak energies, it is necessary to consider the energy loss of the incident electrons in the light-blocking layer and the $\Delta \mathrm{E}$ detector. The Geant 4 simulation estimates that the characteristic peak energies of ${ }^{207} \mathrm{Bi}, 481.6$ $\mathrm{keV}, 976.4 \mathrm{keV}$, and $1048.1 \mathrm{keV}$, are reduced after passing through the light-blocking layer ( $50 \mu \mathrm{m}$ aluminum) and the $\Delta \mathrm{E}$ detector (100 $\mu \mathrm{m}$ silicon), to $424.6 \mathrm{keV}, 930.4 \mathrm{keV}$, and $1003.6 \mathrm{keV}$, respectively. The central ADC channels of the characteristic peaks of $481.6 \mathrm{keV}, 976.4 \mathrm{keV}$, and $1048.1 \mathrm{keV}$ can be derived by Gaussian fitting, resulting in 163.7, 405.8, and 438.7, respectively. Figure 19 shows the linear fitting results of the central ADC channels for the three ${ }^{207} \mathrm{Bi}$ peaks, and the residual energies after passing through the light barrier and the $\Delta \mathrm{E}$ detector. It is evident that the HED sensor head shows good linearity in the energy response, $\sim 0.3 \%$.

The linear fitting result shown in Figure 19 is defined in Equation (2):

$$
E=2.097 \times \mathrm{Ch}+80.85,
$$

where $E$ is the electron energy collected by the detector, $C h$ is the channel number, and 2.097 is the channel width or the energy per channel. According to Equation (2), we can calculate the ADC channel numbers corresponding to the five energy boundaries for 


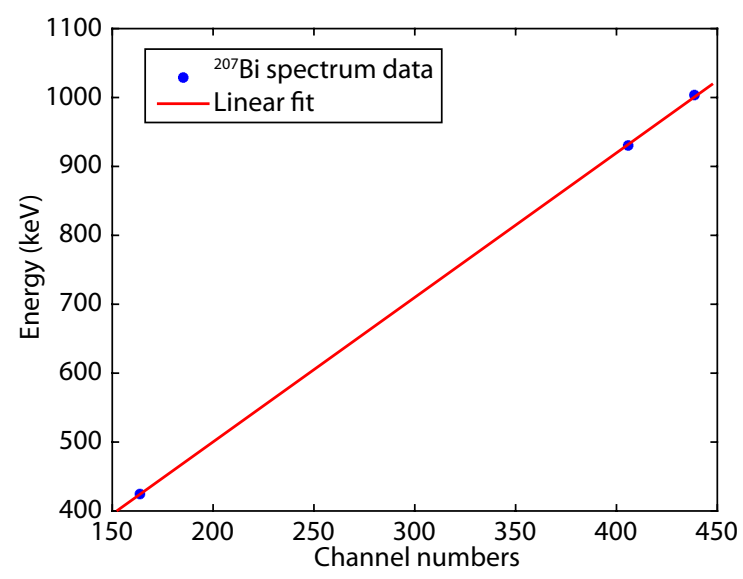

Figure 19. The energy linearity of the HED, with fitting result shown in red. The abscissa is the ADC channel number, and the ordinate is the peak energy in keV. The blue ${ }^{* \prime}$ corresponds to the central channels of the three ${ }^{207} \mathrm{Bi}$ energy peaks at $481.6 \mathrm{keV}, 976.4 \mathrm{keV}$, and $1048.1 \mathrm{keV}$.

the four HED energy bands. These ADC channel numbers are the boundary thresholds sent from the DPU to the HED to derive the counts of the four energy bands.

The test signal source is used to measure the system electronics noise of the $E$ detector. Figure 20 shows the response of the $E$ detector to the test signal, which follows a Gaussian distribution. The FWHM of the response curve can be obtained by Gaussian fitting and is 16.1 ADC channels wide. Based on the energy calibration result, the calculated system electronics noise of the $E$ detector is $33.8 \mathrm{keV}$. Although the noise of the HED is nearly an order of magnitude larger than that of the MES, it fulfills the detection requirement of the HED for 0.5-3 MeV electrons.

The angular response of the HED sensor head was verified by Monte Carlo simulation. Figure 21 shows the angular response results of the HED sensor head to high-energy electrons incident in different directions, as simulated with Geant4. The normalized counts of the $\mathrm{E}$ detector for each directional angle are simulated

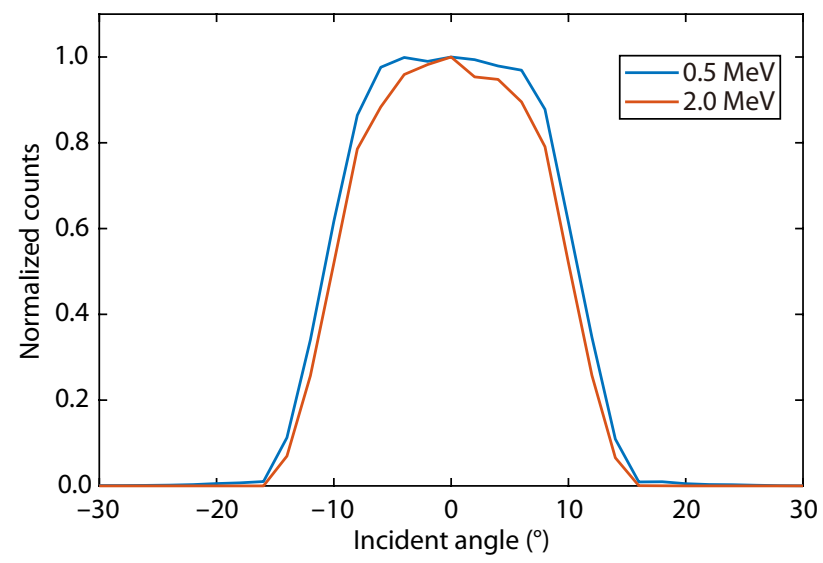

Figure 21. The angular response of the HED to incident electrons. The abscissa is the incident angle, and the ordinate is the normalized counts; $0^{\circ}$ means normal incidence.

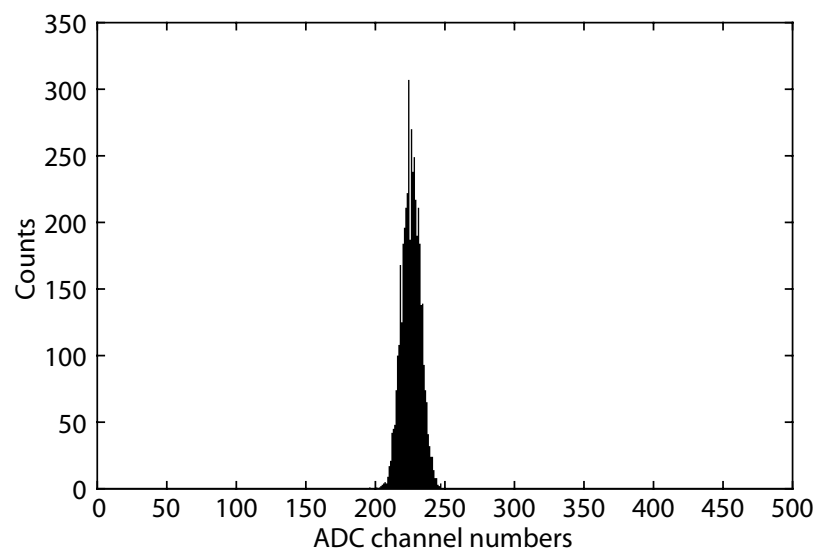

Figure 20. HED response to the test signal. The abscissa is the ADC channel number, and the ordinate is counts.

for incident electrons with energies of $0.5 \mathrm{MeV}$ and 2.0 MeV. The directional responses of the HED sensor head for two single-energy electrons are very similar, and the FWHM of the response curve is about $20-22^{\circ}$ which meets the detection requirements of high-energy electrons.

The geometric factors of each HED energy band are simulated with Geant4, and the results are consistent with those shown in Table 2.

\subsection{DDCM Testing and Calibration}

The DDCM calibration consists of applying a high voltage on the charging sensor to simulate the voltage generated by incident electrons in the inner material, to determine the relationship between the voltage applied to the sensor and the output voltage. The charging voltage of the sensor can be determined according to the linear relationship between the high voltage applied to the sensor and the output voltage of the DDCM amplifier circuit converted by the ADC. Figure 22 shows the linear relationship between the DC high voltage applied to the DDCM and the

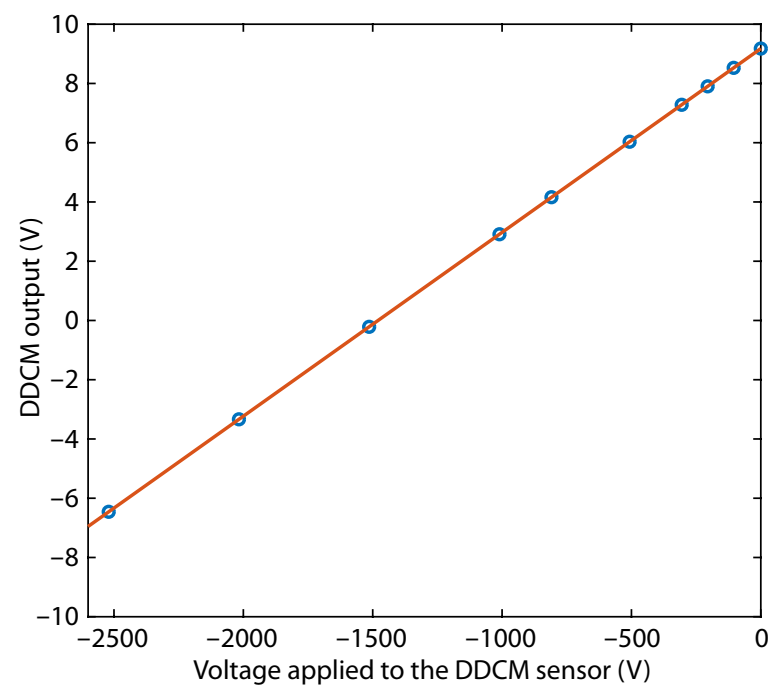

Figure 22. The linear relationship between the voltage applied to the charging voltage sensor of the DDCM and the DDCM output. 
output voltage on the MEO1 satellite.

Figure 22 shows that, for the required range of the charging voltage $(-2500 \mathrm{~V}$ to $0 \mathrm{~V})$, the DDCM output has very good linearity with the applied voltage, better than $0.02 \%$. The linear relationship shown in Figure 22 is defined as:

$$
V_{\text {out }}=H_{V} \times 6.2038 \times 10^{-3}+9.1774 \text {, }
$$

where $V_{\text {out }}$ is the analog voltage output of the DDCM, and $H_{V}$ is the charging voltage. Using Equation (3), the charging voltage of the sensor can be obtained from the DDCM data.

Similarly, by applying a weak current to the surface charging current sensor, the relationship between the sensor current and the circuit output voltage can be determined. Figure 23 shows the linear relationship between the weak current applied to the charging current sensor of the DDCM and the output voltage on the MEO1. The relationship has good linearity, $\sim 0.08 \%$, and the linear function is:

$$
V_{\text {out }}=0.36583 \times I+9.6592
$$

The charging current can be calculated with DDCM data using Equation (4).

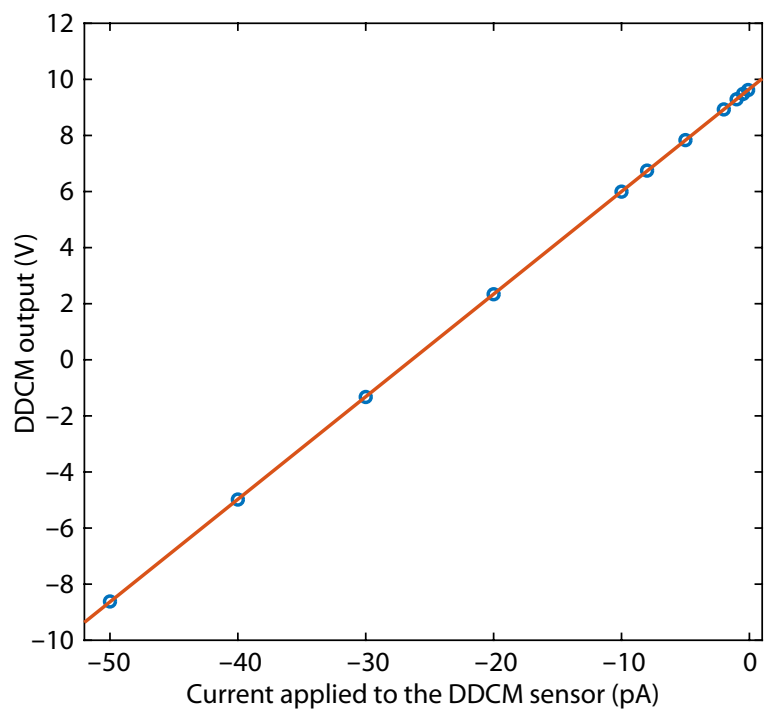

Figure 23. The linear relationship between the current applied to the charging current sensor of the DDCM and the DDCM output.

An electron gun system is used to calibrate the DDCM deep charging current measurements; the detailed results were described by Yu XQ et al. (2020). The calibration results indicate that the DDCM meets the requirements for deep dielectric charging monitoring.

\section{Initial Results}

\subsection{Energy spectra comparison with MagEIS}

The EEDP systems on MEO1 and MEO2 have been in orbit for nearly two years, and the EEDP on MEO3 has also operated in orbit normally for nearly one year. Since the data sampling rates of
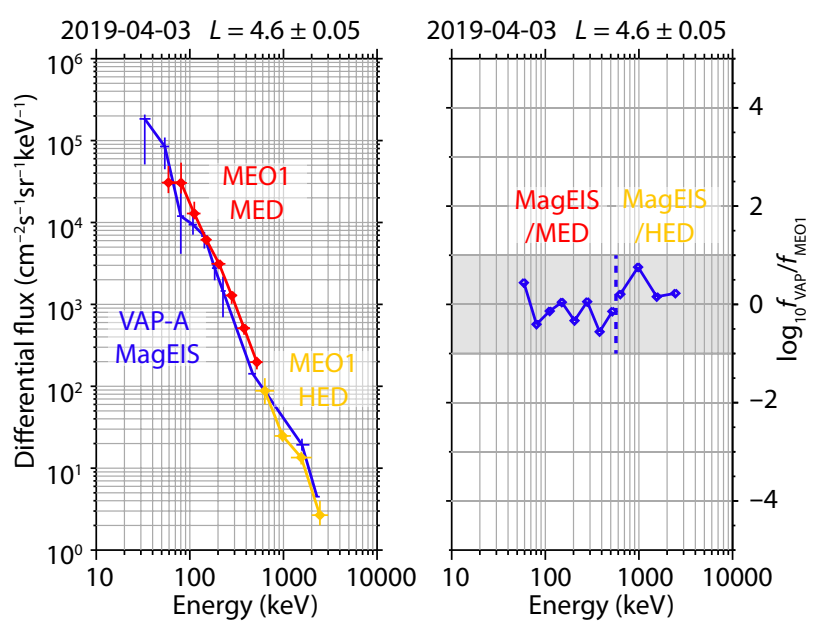

Figure 24. Comparison of the daily averaged electron spectra observed by the MagEIS on board VAP-A with the MES and HED on MEO1 at $L=4.6 \pm 0.05$ on April 3, 2019. The curve of the MES is the omnidirectional electron spectrum.

these instruments are $1 \mathrm{~s}$, a large amount of data has been accumulated. The electron energy spectra measured by MEO1 are compared with those measured by MagEIS on board VAP-A to verify the validity of the MES and HED data. Figure 24 compares the daily averaged electron energy spectra observed by MagEIS at $L=4.6 \pm 0.05$ on April 3, 2019 (a relatively quiet geomagnetic period, daily average Dst -18.9 nT) with spectra observed by MEO1. The relative deviation of the energy spectra is also shown in Figure 24. At this $L$-shell, the locations of VAP-A and MEO1 are close to the geomagnetic equator at different longitude. Fortunately, the longitudinal variation of electron flux in the energy range of $50 \mathrm{keV}$ 3.0 MeV is not significant during this geomagnetic quiet period.

As shown in Figure 24, the electron energy spectra observed by the MES and HED on MEO1 are in good agreement with MagEIS. The average relative deviation of the energy spectra is about $27.3 \%$. The electron energy spectra obtained by the MES and HED exhibit a similar power-law distribution.

The electron spectra observed by the MES and HED on MEO1, $\mathrm{MEO} 2$, and MEO3 in the same period and L-shell, are compared to confirm the consistency of electron detection by the three EEDPs. Figure 25 shows the daily averaged electron flux at $L=4.6 \pm 0.05$ on January 14, 2020 (a quiet geomagnetic period, daily average Dst $>-2.9 \mathrm{nT}$ ) measured by MEO1, MEO2, and MEO3. The results obtained from the instruments have good consistency. The electron flux measured by the HED on MEO3 is slightly higher than that measured by MEO1 and MEO2. This discrepancy is related to the different orbital plane of $\mathrm{MEO} 3$ compared to the other two satellites.

The results in Figure 24 and Figure 25 indicate that the electron energy spectra measured by the MES and HED on board the three MEO Chinese navigation satellites show a good power-law distribution and are in good agreement with MagEIS on VAP-A. In addition, the measurements of the instruments on the three MEO satellites are highly consistent, demonstrating overall reliability of 


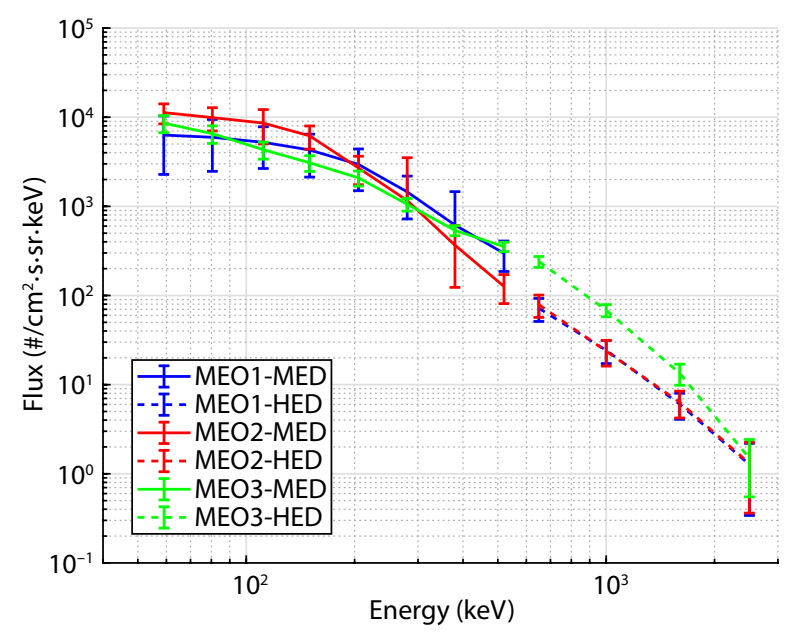

Figure 25. The daily averaged electron spectra observed by the MES and HED of the EEDPs on board MEO1, MEO2, and MEO3 at $L=4.6 \pm$ 0.05 on Jan 14, 2020.

the EEDP particle instruments.

\subsection{Three-belt Structure}

An important discovery of VAP was the three-belt structure of the electron radiation belts described in the introduction. However, due to the altitude of the orbit's apogee, VAP could only observe the regions with $L \leq 6$ but not the outer boundary of the new belt of high-energy electrons. Observations by the MEO Chinese navigation satellites can compensate for VAP's coverage shortcomings. Figure 26 shows the newly generated belt of high-energy electrons observed in the outer belt by the HED on MEO1 at the end of November 2019. Since the beginning of November, the high-energy electrons at the outer boundary of the outer belt generated in the last event had been declining, and the original outer belt boundary decayed to the region near $L=4$ on Nov 22 . Subsequently, due to a small magnetic storm that occurred around Nov 21, a new electron belt began to appear in the region of $L=5-6$ and gradually strengthened as its outer boundary extended to $L \sim 8$. The inner boundary of the new belt was clearly distinguishable from the old one, especially during Nov 22-25. In addition, the HED observations show that the new electron belt was measurable at $<2.0 \mathrm{MeV}$ electron flux but more difficult to distinguish in the 2.0-3.0 MeV electron flux. The HED then observed the complete generation and development of a new electron belt on November 22, 2019, filling the gap of the VAP observation. However, due to the orbit limitation of the MEO Chinese navigation satellites, only regions with $L \geq 4.4$ can be observed. Therefore, there was not enough evidence so additional data observations were needed.

Measurements by the particle radiation detector (PRD) on board the CBERS-1/04 spacecraft support that the radiation belt electrons formed a three-belt structure on November 22, 2019. CBERS$1 / 04$ has a sun-synchronous orbit with an altitude of $780 \mathrm{~km}$ and an orbital period of about $100 \mathrm{~min}$. The satellite is equipped with the PRD developed by Peking University that can measure 0.5-2.0 $\mathrm{MeV}$ and $>2.0 \mathrm{MeV}$ electron footprints for a large range of $L$-shells (Chen $Z$ et al., 2017). Figure 27 shows the temporal and $L$-shell dis- tributions of the $0.5-2.0 \mathrm{MeV}$ electron flux observed by the particle detector on CBERS-1/04 and the 1.2-2.0 MeV electron flux obtained from the HED on MEO1 in November 2019. Due to the low altitude of CBERS-1/04, the geomagnetic field intensity is significantly affected by geomagnetic anomalies, resulting in a significant difference in the height of the geomagnetic mirror point at $780 \mathrm{~km}$. Therefore, the electron fluxes observed at varying longitudes in the same $L$-shell at $780 \mathrm{~km}$ are quite different (Zou H et al., 2006a) and only the daily average electron fluxes in the $L$-shell observed by the particle detector on CBERS-1/04 can be displayed. The sampling rate of the particle detector is $12 \mathrm{~s}$; thus, the resolution of the temporal and $L$-shell observations is relatively low. The MEO observations provide significantly higher temporal resolution and $L$-shell distribution, as shown in Figure 27.

The results of the particle detector observations in Figure 27 show that during November 2019, the slot region boundary between the outer belt and the inner belt was well defined, and no slot penetration event occurred. After November 22, a slight increase in the electron flux was observed in the high $L$-shell region of the outer belt. However, due to the insufficient $L$-shell resolution of the CBERS-1/04 observations, the boundary between the old and the new electron belt in the outer belt cannot be distinguished. In contrast, the HED on MEO1 has a very high L-shell and temporal resolution; a new electron belt was observed in the $L$-shell range of 5.5-7 on November 22. and the boundary between the new belt and the old one is well-defined. Thus, the high-energy electron observations of the particle detector on CBERS-1/04 and MEO1 together indicate the occurrence of a three-belt structure in the radiation belt region in November. This is the first report of the three-belt structure observed by the particle detectors on MEO1.

\subsection{Derived Pitch Angle Distribution of Energetic Electrons}

Since the MES uses pinhole imaging technology, the electron fluxes can be measured simultaneously from nine directions with a $180^{\circ}$ viewing angle, which means the MES observation has a high angular resolution. There is no scientific payload on the satellites to measure the geomagnetic field; therefore, accurate pitch angle information is not available. However, since the geomagnetic field intensity in MEO is much stronger (in some regions it can reach $1000 \mathrm{nT}$ ) than that in GEO, the geomagnetic field model IGRF plus OP77Q (Olson and Pfitzer, 1977) can be used to calculate the direction of the magnetic field corresponding to the MEO at the satellites' locations. Subsequently, the pitch angles of the MES nine observational directions can be determined according to the orientation of the sensor. Figure 28 shows the pitch angles for eight energy bands of medium-energy electrons measured by the MES on MEO3 at $L=4.6 \pm 0.05$ during a geomagnetic calm period (average Dst > $-2.8 \mathrm{nT}$ ) on February 10, 2020.

The maximum flux for the eight energy bands of medium-energy electrons observed by the MES on MEO3 occurs at a pitch angle range of $70^{\circ}-100^{\circ}$, while the fluxes are relatively low close to $0^{\circ}$ and $180^{\circ}$. This result is in agreement with the theoretical pitch angle near the geomagnetic equator in the outer belt and VAP observations (Li and Hudson, 2019), though the MES resolution is 
(a)

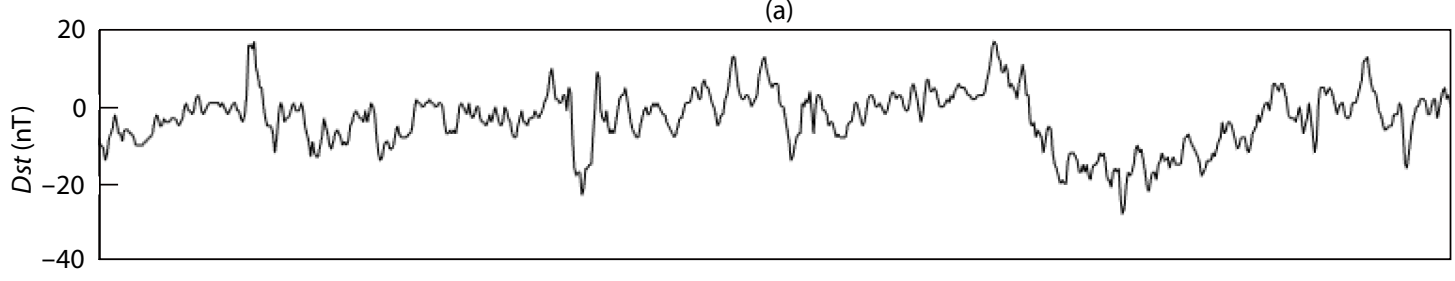

(b)

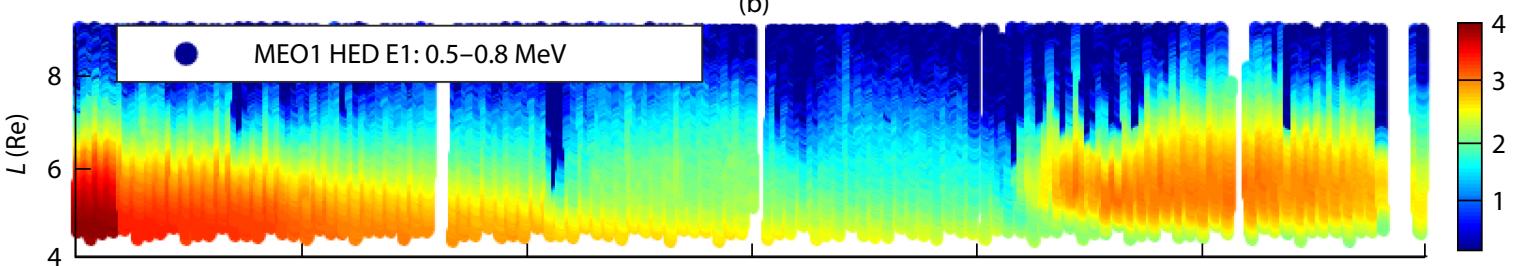

(c)

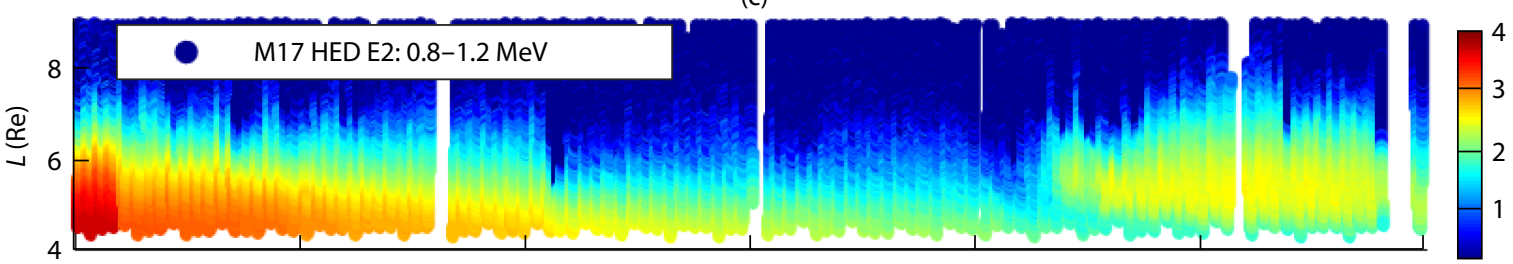

(d)

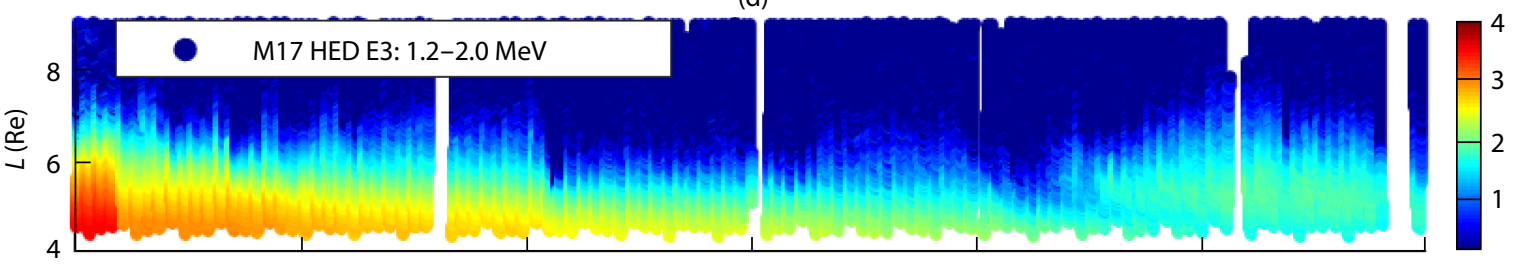

(e)

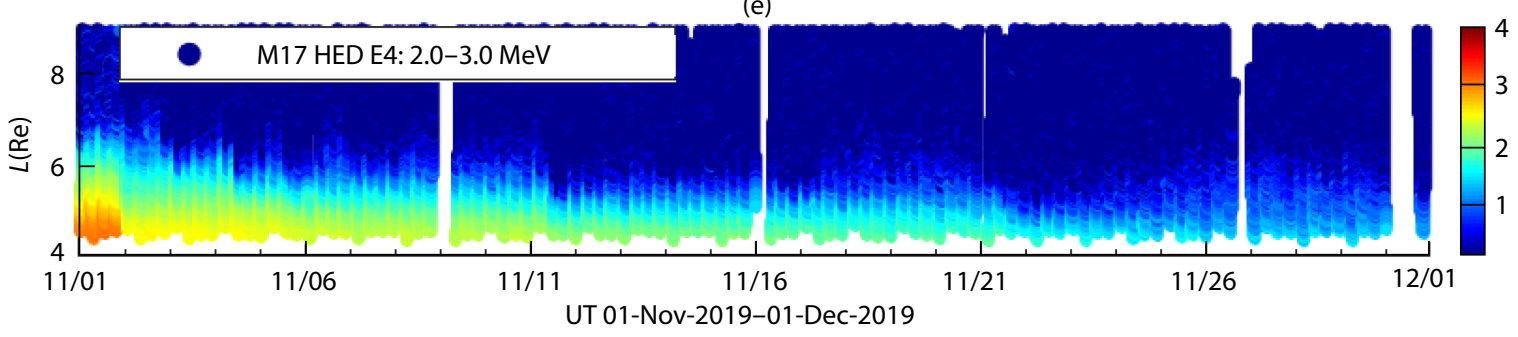

Figure 26. The temporal and $L$-shell distributions of four high-energy electron channels observed by the HED on MEO1 in November 2019. Panel (a) shows the temporal variation of the Dst index. Panels $(\mathrm{b}-\mathrm{e})$ show the temporal and $L$-shell distributions of the electron fluxes at $0.5-0.8 \mathrm{MeV}$, 0.8-1.2 MeV, 1.2-2.0 MeV, and 2.0-3.0 MeV respectively.

not as high as VAP. Since the MEO Chinese navigation satellite is a three-axis stabilized platform, the pitch angle values for mediumenergy electrons measured by the MES are acceptable, and this is the first report of such a measurement by a particle detector developed in China.

\subsection{Results of Electron Detection and Deep Charging Detection During Geomagnetic Storms}

Current research (Fennell et al., 2000) has shown that deep charging events are usually related to high-energy electron bursts, and high electron flux can persist for prolonged periods. The highenergy electron flux variation measured by the HED and the deep charging observations of the DDCM on MEO1 were investigated during geomagnetic storms from August to September 2019, durich which three small geomagnetic storms occurred every 27 days (minimum Dst $\sim-50 n T$ ). Figure 29 shows the temporal and $L$ - shell distributions of high-energy electron fluxes and temporal variation of the Dst index in the same period. The HED observations showed three high electron flux events in the outer belt corresponding to the recurring geomagnetic storms. A comparison of the two geomagnetic storms with similar Dst indices in August and September shows slightly different electron fluxes in each case; fluxes in September were higher than those in August, and the difference was more pronounced in the higher energy bands.

Figure 30 shows the temporal variations of charging currents and charging voltages measured by the DDCM on MEO1 in the same period as the observations shown in Figure 29. A comparison of Figure 29 and Figure 30 indicates that the temporal variations measured by the DDCM are consistent with those of the high-energy electron fluxes. The charging current of the first layer of the DDCM sensor head increased significantly in the two magnetic storms in August and September, but the charging current in the 
(a)

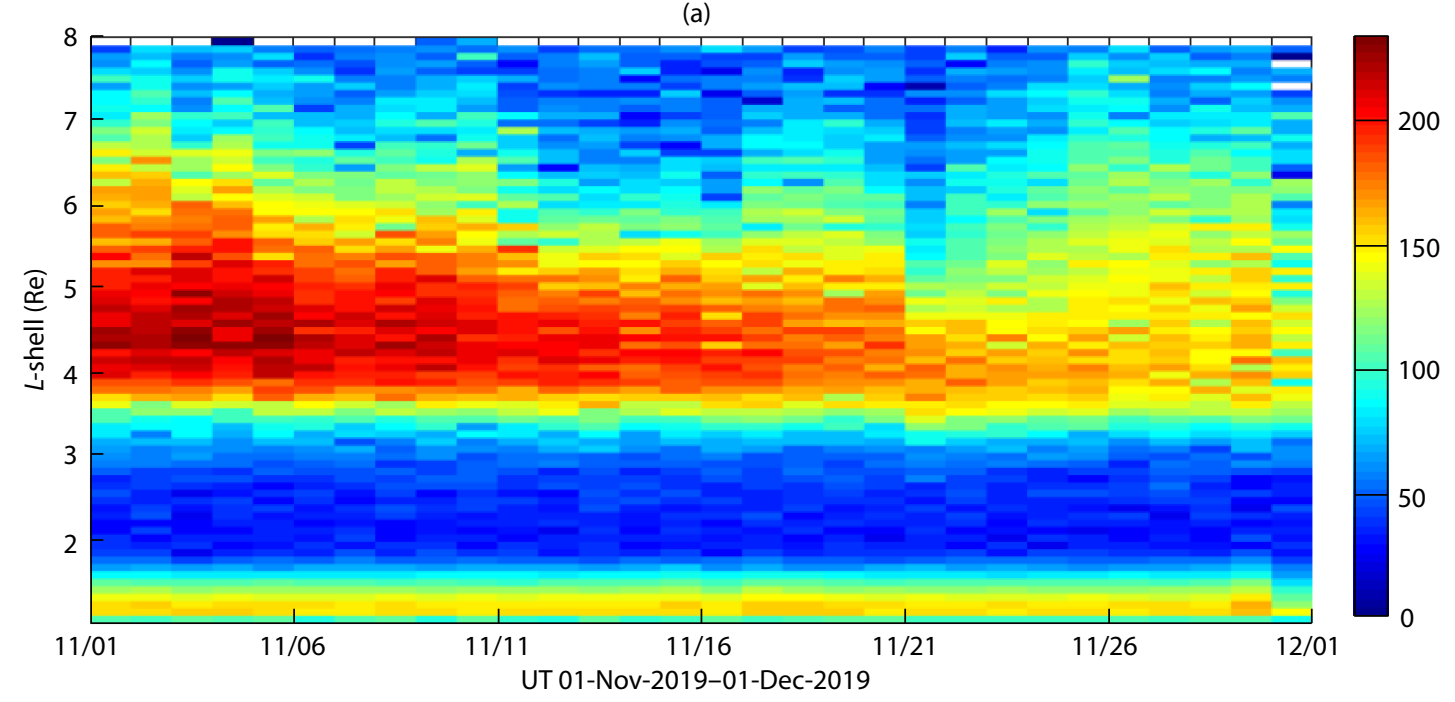

(b)

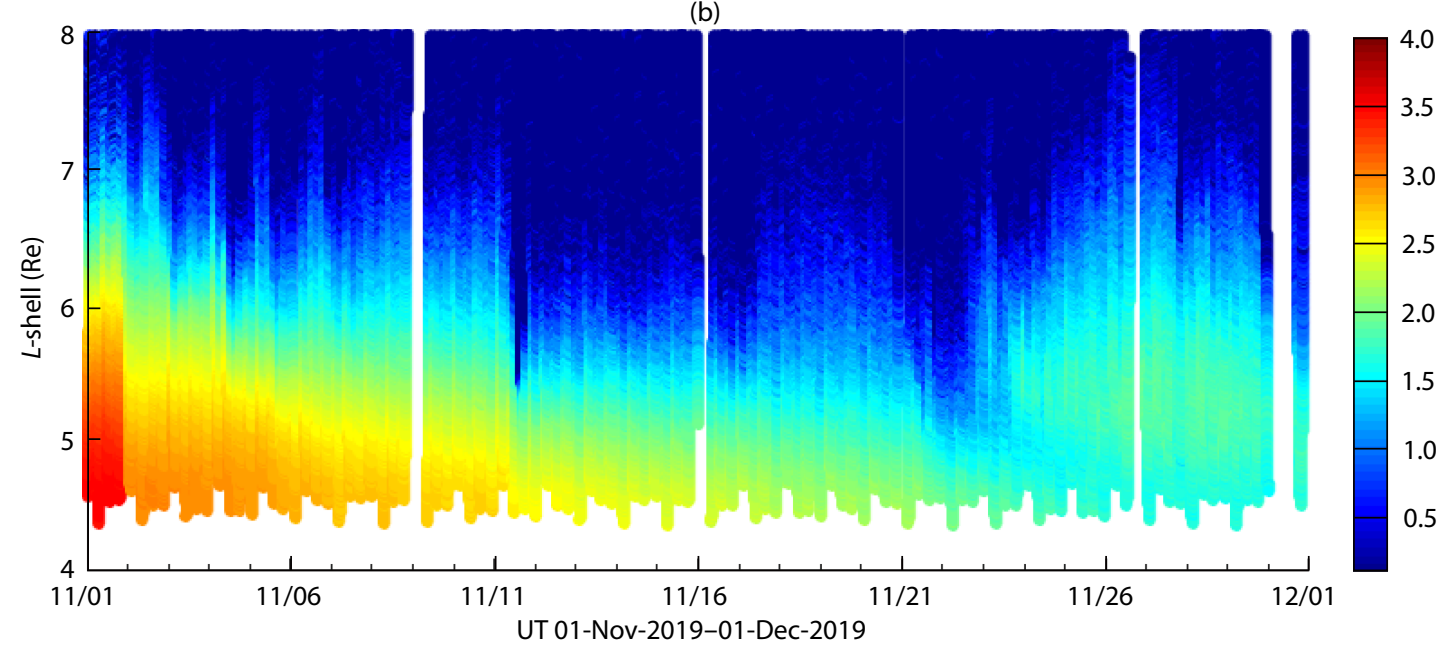

Figure 27. The temporal and $L$-shell distributions of $0.5-2.0 \mathrm{MeV}$ electrons observed by the particle radiation monitor on CBERS-1/04, showing (a) the daily averaged electron flux measured by CBERS-1/04, and (b) flux of 1.2-2.0 MeV electrons observed by the HED MEO1 in November 2019.

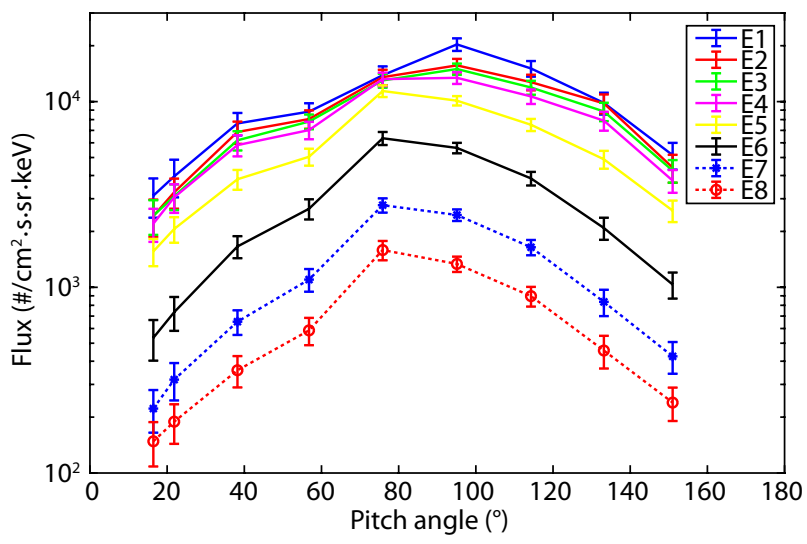

Figure 28. The electron pitch angles for 8 channels of the MES on the MEO3 satellite at $L=4.6$ on February 10,2020 .

September storm was significantly higher than the August storm. The charging current of the fourth layer showed a negligible response to the storm in August with a significant increase in the
September storm, while the charging voltage only showed a significant increase in the September storm. The difference in the DDCM observations during the two geomagnetic storms is believed to be caused by the difference in high-energy electron flux enhancement, as the MeV electron flux in the September storm was several times higher than the August storm. The MeV electrons have stronger penetrating ability and can penetrate the sensor surface and are deposited more deeply, thus, the charging currents and voltages are larger. This result shows that the DDCM observations are in agreement with the high-energy electron observations of the HED.

\section{Discussion and Summary}

This paper has described in detail the basic principles, design scheme, and results of ground tests and calibration, as well as preliminary observations of the EEDP developed by the scientific space payload team at Peking University. The EEDP includes the MES, which is similar to the BD-IES developed by the same team at Peking University, as well as the HED and DDCM. The BD-IES mounted on the Chinese navigation satellite in IGSO has oper- 
(a)

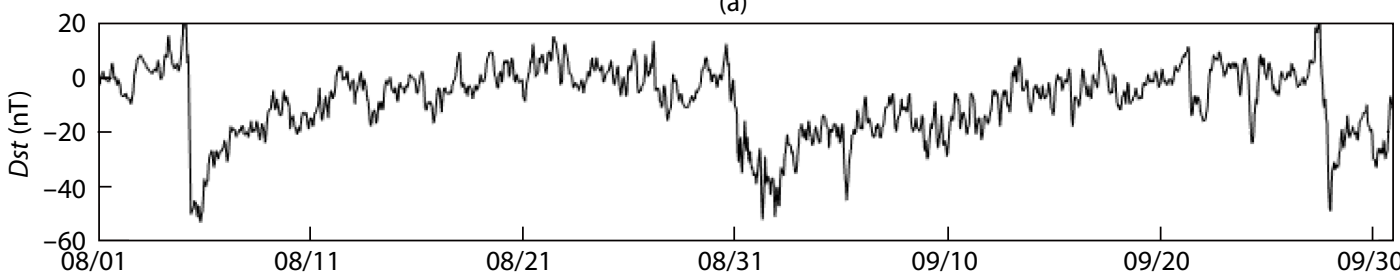

(b)

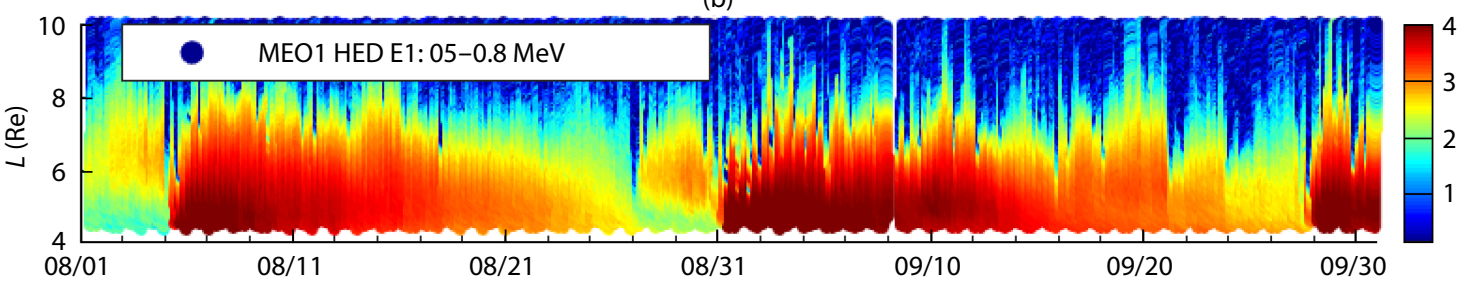

(c)

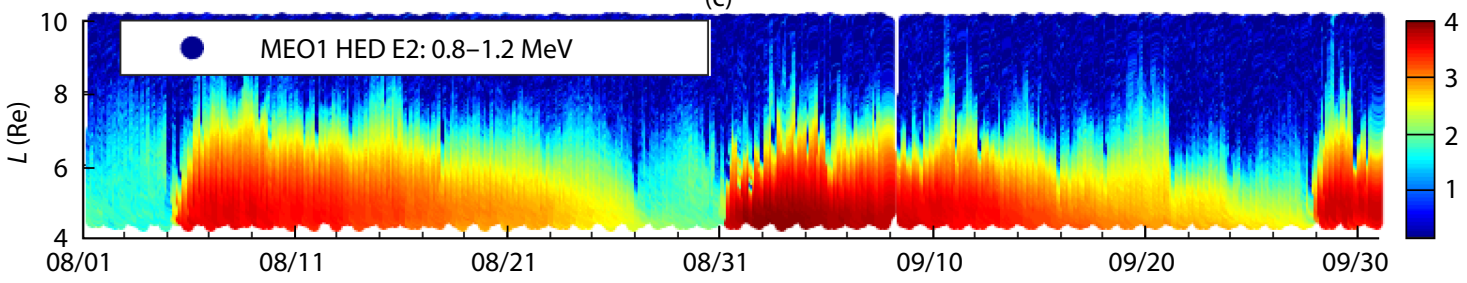

(d)

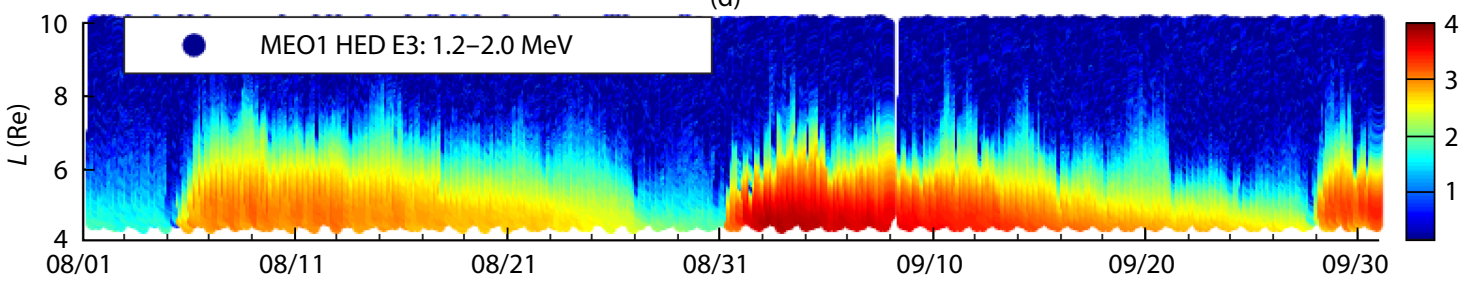

(e)

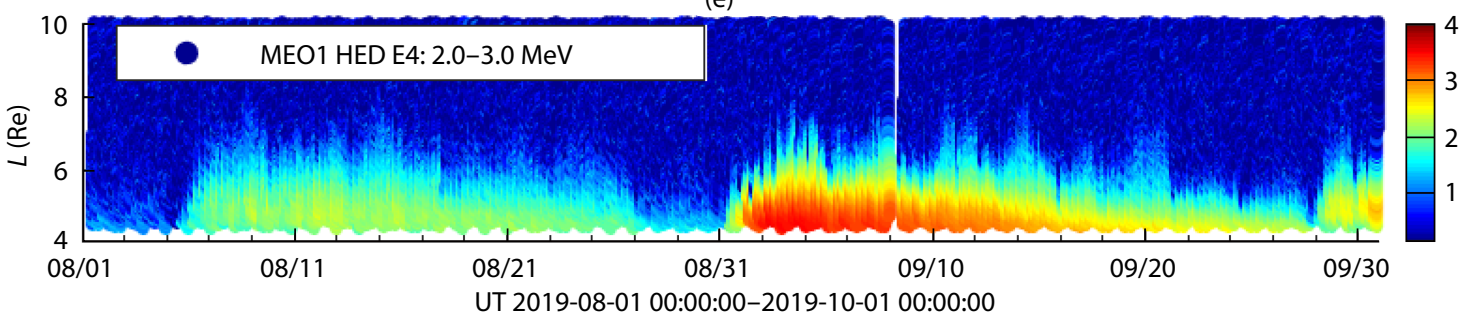

Figure 29. The temporal and $L$-shell distributions of high-energy electron fluxes measured by the HED on MEO1 during storms in August and September, 2019. Panel (a) shows the time series of the Dst index. Panels (b-e) show the fluxes for 0.5-0.8 MeV, 0.8-1.2 MeV, 1.2-2.0 MeV, and 2.0-3.0 MeV respectively. The electron differential flux is shown on a logarithmic scale in \#/cm².s.sr.keV.

ated normally for over five years. The data quality was verified, and important results were achieved regarding substorm injection, wave-particle interaction, drifting electron holes, solar wind energy particles, and responses to corotating interaction region (CIR) events (Zong QG et al., 2016; Li L et al., 2017a, b; Wang LH et al., 2017; Liu ZY et al., 2018, 2019; Yin ZF et al., 2019; Chen XR et al., $2020 a, b)$. In this study, the ground test and calibration results verified that the performance of the MES was at the level of the BD-IES. The high-energy particle detectors developed by Peking University have been successively deployed on CBERS-1, and many results were obtained (Xiao Z et al., 2003; Zou H et al., 2004, 2006a, b, 2007; Hao YQ et al., 2007; Chen Z et al., 2017).

Three EEDPs are currently deployed on three Chinese MEO navigation satellites. Their observations cover a large $L$-shell range (the region of $L>4.4$ from the center of the outer belt to the outer boundary), which partially overlaps with VAP observations. Therefore, the data quality of the MES and HED were verified by comparing the electron energy spectra observed by the Chinese MEO navigation satellites and VAP for the same period and $L$-shell. Furthermore, the EEDP observations compensate for the shortcoming of VAP insufficient coverage of the outer radiation belt. The EEDP can observe the response of the outer boundary of the outer radiation belt to solar wind shock waves, CMEs, and other burst events with high spatial and temporal resolution.

At present, MEO is a crucial orbit for navigation and GPS satellite systems in many countries. Strong geomagnetic storms caused by large solar storms can cause malfunctions or failures of important satellite payloads, or even the loss of satellites, which may adversely affect the stability or interrupt service systems. Therefore, there is an urgent need for continuous high-temporal resolution 

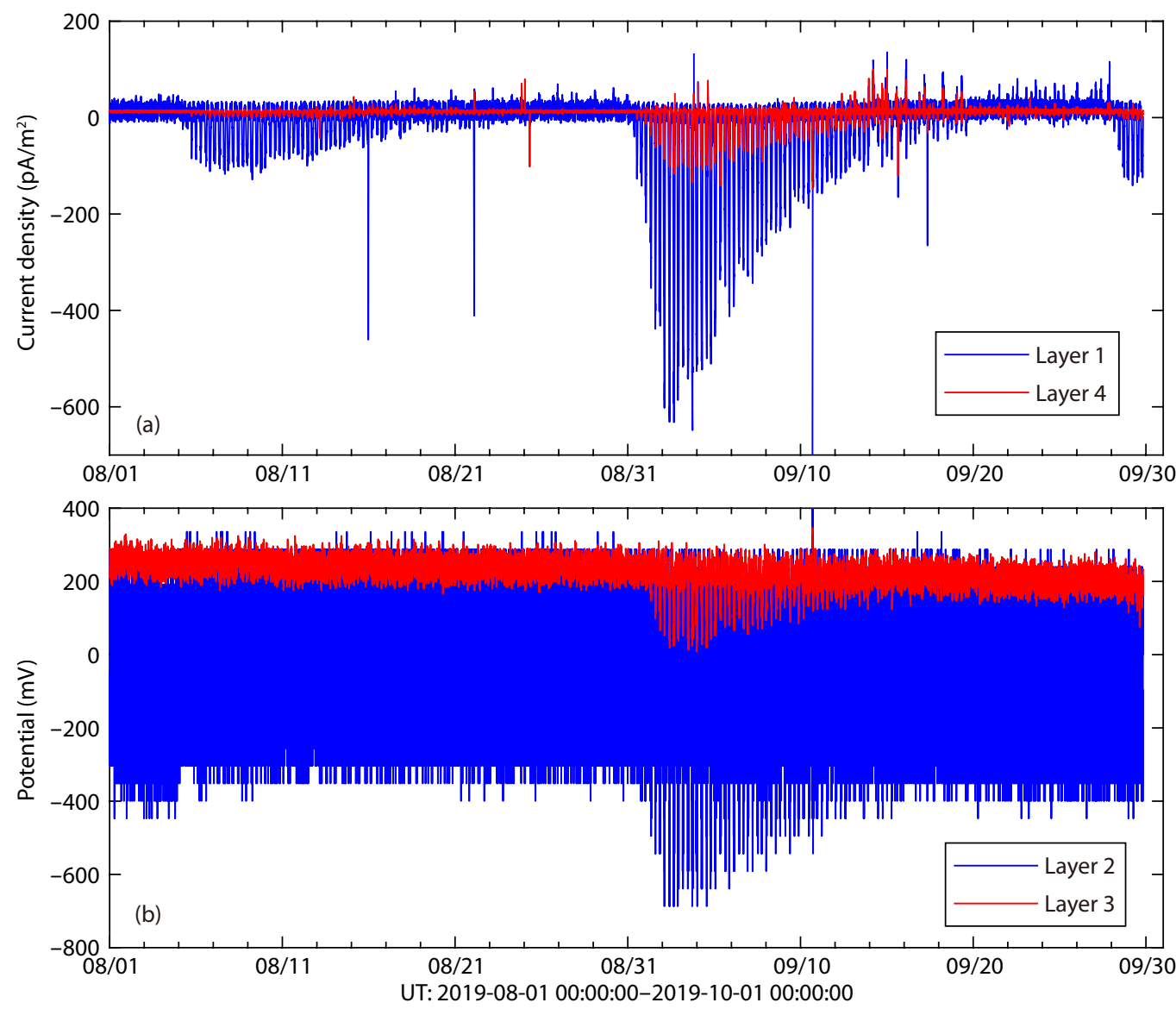

Figure 30. Temporal variations of charging currents and potentials measured by the DDCM on MEO1 during storms in August and September 2019. Panel (a) shows the time series of charging currents for layer 1 and layer 4 . Panel (b) shows the time series of charging potentials for layer 2 and layer 3.

monitoring of space weather in MEO. After the end of the VAP mission, the only other remaining radiation belt scientific research satellite in MEO is the Japanese ERG mission (Miyoshi et al., 2015), a single satellite designed to conduct joint observations with VAP and ground-based assets. Going forward, the EEDP on the three Chinese MEO navigation satellites will play an important role in space science research and space weather applications. The preliminary results of the EEDP demonstrate sufficiently high data quality of the instrument for this important task.

The FY-3/05 satellite in LEO and the FY-4/02 satellite in GEO are scheduled to be launched in 2022. At that time, the energetic electron detectors developed by Peking University will be on board three MEO satellites, an IGSO satellite, a GEO satellite, and an LEO satellite as shown in Figure 31. This six-constellation observation network will provide global high-resolution observations of the radiation belts. With the approach of the next solar maximum, this constellation will enable advanced Chinese radiation belt research and space weather monitoring.

\section{Acknowledgment}

The authors sincerely thank the scientific payload development team at Peking University, the collaborators involved in instrument data analysis, and all personnel who contributed efforts to the successful launches and operations of the instruments. This

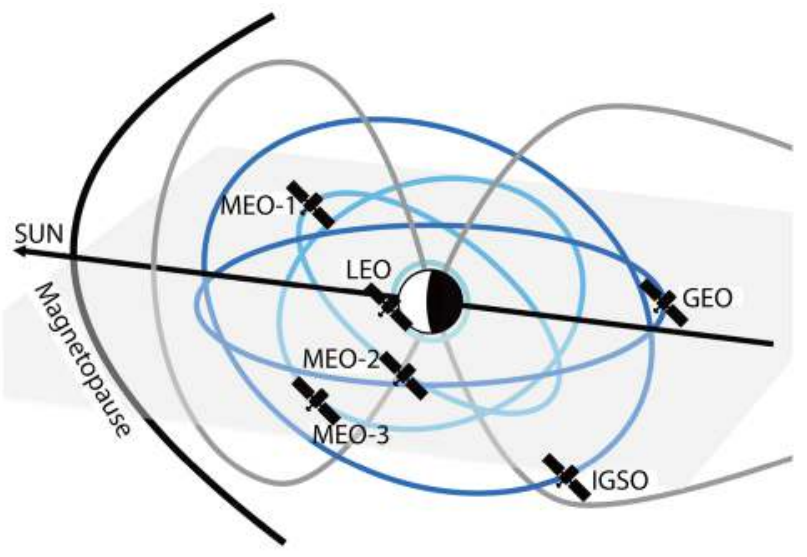

Figure 31. The six-satellite constellation observation network of energetic electron detection in the magnetosphere, developed by Peking University.

research was supported by the National Natural Science Foundation of China (No. 41374167, 41421003, 41474140) and China's National Basic Research and Development Program (No. 2012CB825603). The geomagnetic and solar wind data were obtained from the space data section of the NASA website (http://spdf.gsfc.nasa.gov/). The authors thank D. Boscher, S. Bourdarie, P. O'Brien, and T. Guild for providing the ONERA-DESP 
library V4.2, Toulouse-France, 2004-2008, available at http:// craterre.onecert.fr/support/user_guide.html.

\section{References}

Baker, D. N., Kanekal, S. G., Li, X., Monk, S. P., Goldstein, J., and Burch, J. L. (2004). An extreme distortion of the Van Allen belt arising from the 'Hallowe'en' solar storm in 2003. Nature, 432(7019), 878-881. https://doi.org/10.1038/nature03116

Baker, D. N., Kanekal, S. G., Hoxie, V. C., Batiste, S., Bolton, M., Li, X., Elkington, S. R., Monk, S., Reukauf, R., ... Friedel, R. (2013a). The Relativistic ElectronProton Telescope (REPT) instrument on board the Radiation Belt Storm Probes (RBSP) spacecraft: characterization of Earth's radiation belt highenergy particle Populations. Space Sci. Rev., 179(1-4), 337-381. https://doi.org/10.1007/s11214-012-9950

Baker, D. N., Kanekal, S. G., Hoxie, V. C., Henderson, M. G., Li, X., Spence, H. E., Elkington, S. R., Friedel, R. H. W., Goldstein, J., ... Claudepierre, S. G. (2013b). A long-lived relativistic electron storage ring embedded in earth's outer Van Allen belt. Science, 340(6129), 186-190. https://doi.org/10.1126/science.1233518

Baker, D. N., Erickson, P. J., Fennell, J. F., Foster, J. C., Jaynes, A. N., and Verronen, P. T. (2018). Space weather effects in the Earth's radiation belts. Space Sci. Rev., 214, 17. https://doi.org/10.1007/s11214-017-0452-7

Baker, D. N., Hoxie, V., Zhao, H., Jaynes, A. N., Kanekal, S., Li, X. L., and Elkington, S. (2019). Multiyear measurements of radiation belt electrons: acceleration, transport, and loss. J. Geophys. Res. Space Phys., 124(4), 2588-2602. https://doi.org/10.1029/2018JA026259

Blake, J. B., Kolasinski, W. A., Fillius, R. W., and Mullen, E. G. (1992). Injection of electrons and protons with energies of tens of $\mathrm{MeV}$ into $\mathrm{L}<3$ on 24 March 1991. Geophys. Res. Lett., 19(8), 821-824. https://doi.org/10.1029/92GL00624

Blake, J. B., Fennell, J. F., Friesen, L. M., Johnson, B. M., Kolasinski, W. A., Mabry, D. J., Osborn, J. V., Penzin, S. H., Schnauss, E. R., ... Hall, D. (1995). CEPPAD: comprehensive energetic particle and pitch angle distribution experiment on POLAR. Space Sci. Rev., 71(1-4), 531-562. https://doi.org/10.1007/BF00751340

Blake, J. B., Carranza, P. A., Claudepierre, S. G., Clemmons, J. H., Crain, W. R. Jr., Dotan, Y., Fennell, J. F., Fuentes, F. H., Galvan, R. M., ... Zakrzewski, M. P. (2013). The Magnetic electron ion spectrometer (MagEIS) instruments aboard the radiation belt storm probes (RBSP) spacecraft. Space Sci. Rev., 179(1-4), 383-421. https://doi.org/10.1007/s11214-013-9991-8

Bortnik, J., Chu, X. N., Ma, Q. L., Li, W., Zhang, X. J., Thorne, R. M., Angelopoulos, V., Denton, R. E., Kletzing, C. A., ... Baker, D. N. (2018). Artificial neural networks for determining magnetospheric conditions. In E. Camporeale, et al. (Eds.), Machine Learning Techniques for Space Weather (pp. 279-300). Amsterdam: Elsevier. https://doi.org/10.1016/B978-0-12-811788-0.00011-1

Chen, X. R., Zong, Q. G., Zou, H., Wang, Y. F., Zhou, X. Z., and Biake, J. B. (2020a). Distribution of energetic electrons in the near earth space: new observations from the BeiDa Imaging Electron Spectrometer and the Van Allen Probes. Planet. Space Sci., 186, 104919. https://doi.org/10.1016/j.pss.2020.104919

Chen, X. R., Zong, Q. G., Zou, H., Zhou, X. Z., Li, L., Hao, Y. X., and Wang Y. F. (2020b). BeiDa imaging electron spectrometer observation of multi-period electron flux modulation caused by localized ultra-low-frequency waves. Ann. Geophys., 38(4), 801-813. https://doi.org/10.5194/angeo-38-801-2020

Chen, Z., Chen, H. F., Li, Y. F., Xiang, H. W., Yu, X. Q., Shi, W. H., Hao, Z. H., Zou, H., Zou, J. Q., and Zhong, W. Y. (2017). Variations of the relativistic electron flux after a magnetospheric compression event. Sci. China Tech. Sci., 60(4), 638-647. https://doi.org/10.1007/s11431-016-9008-3

Evans, D. S., and Greer, M. S. (2004). Polar orbiting environmental satellite space Environment monitor-2: Instrument descriptions and archive data documentation. Tech Memo version 1.3, Boulder: US Department of Commerce, National Oceanic and Atmospheric Administration, Oceanic and Atmospheric Research Laboratories, Space Environment Center.

Fennell, J. F., Koons, H. C., Chen, M. W., and Blake, J. B. (2000). Internal charging: a preliminary environmental specification for satellites. IEEE Trans. Plasma Sci., 28(6), 2029-2036. https://doi.org/10.1109/27.902230

Fennell, J. F., Claudepierre, S. G., Blake, J. B., O'Brien, T. P., Clemmons, J. H., Baker,
D. N., Spence, H. E., and Reeves, G. D. (2015). Van Allen Probes show that the inner radiation zone contains no MeV electrons: ECT/MagEIS data. Geophys. Res. Lett., 42(5), 1283-1289. https://doi.org/10.1002/2014GL062874

Frederickson, A. R., Mullen, E. G., Brautigam, D. H., Kerns, K. J., Robinson, P. A., and Holman, E. G. (1991). Radiation-induced insulator discharge pulses in the CRRES internal discharge monitor satellite experiment. IEEE Trans. Nucl. Sci., 38(6), 1614-1621. https://doi.org/10.1109/23.124153

Frederickson, A. R., Holeman, E. G., and Mullen, E. G. (1992). Characteristics of spontaneous electrical discharging of various insulators in space radiations. IEEE Trans. Nucl. Sci., 39(6), 1773-1782. https://doi.org/10.1109/23.211366

Frederickson, A. R. (1996). Method for estimating spontaneous pulse rate for insulators inside spacecraft. IEEE Trans. Nucl. Sci., 43(6), 2778-2782. https://doi.org/10.1109/23.556866

Hao, Y. Q., Xiao, Z., Zou, H., and Zhang, D. H. (2007). Energetic particle radiations measured by particle detector on board CBERS-1 satellite. Chin. Sci. Bull., 52(5), 665-670. https://doi.org/10.1007/s11434-007-0087-2

Hao, Y. X., Zong, Q. G., Zhou, X. Z., Zou, H., Rankin, R., Sun, Y. X., Chen, X. R., Liu, Y., Fu, S. Y., ... Baker, D. N. (2020). A short-lived three-belt structure for sub$\mathrm{MeV}$ electrons in the Van Allen belts: time scale and energy dependence. J. Geophys. Res. Space Phys., 125(9), e2020JA028031. https://doi.org/10.1029/2020JA028031

Johnson, M. H., and Kierein, J. (1992). Combined Release and Radiation Effects Satellite (CRRES): spacecraft and mission. J. Spacecr. Rockets., 29(4), 556-563. https://doi.org/10.2514/3.55641

Leach, R. D., and Alexander, M. B. (1995). Failures and anomalies attributed to spacecraft charging. NASA Reference Publication 1375, Huntsville: NASA.

Li, L., Zhou, X. Z., Zong, Q. G., Chen, X. R., Zou, H., Ren, J., Hao, Y. X., and Zhang, X. G. (2017a). Ultralow frequency wave characteristics extracted from particle data: application of IGSO observations. Sci. China Technol. Sci., 60(3), 419-424. https://doi.org/10.1007/s11431-016-0702-4

Li, L., Zhou, X. Z., Zong, Q. G., Rankin, R., Zou, H., Liu, Y., Chen, X. R., and Hao, Y. $X$. (2017b). Charged particle behavior in localized ultralow frequency waves: theory and observations. Geophys. Res. Lett., 44(12), 5900-5908. https://doi.org/10.1002/2017GL073392

Li, W., and Hudson, M. K. (2019). Earth's Van Allen radiation belts: from discovery to the Van Allen Probes era. J. Geophys. Res. Space Phys., 124(11), 8319-8351. https://doi.org/10.1029/2018JA025940

Liu, Z. Y., Zong, Q. G., Hao, Y. X., Liu, Y., and Chen, X. R. (2018). The radial propagation characteristics of the injection front: a statistical study based on BD-IES and Van Allen probes observations. J. Geophys. Res. Space Phys., 123(3), 1927-1937. https://doi.org/10.1002/2018JA025185

Liu, Z. Y., Zong, Q. G., Zou, H., Wang, Y. F., and Wang, B. (2019). Drifting electron holes occurring during geomagnetically quiet times: BD-IES observations. J. Geophys. Res. Space Phys., 124(11), 8695-8706. https://doi.org/10.1029/2019JA027194

Luo, L., Zou, H., Zong, Q. G., Wang, L. H., Chen, H. F., Shi, W. H., and Yu, X. Q. (2015). Anti-proton contamination design of the imaging energetic electron spectrometer based on Geant4 simulation. Sci. China Technol. Sci., 58(8), 1385-1391. https://doi.org/10.1007/s11431-015-5863-7

Mann, I. R., Ozeke, L. G., Murphy, K. R., Claudepierre, S. G., Turner, D. L., Baker, D. N., Bae, I. J., Kale, A., Milling, D. K., ... Honary, F. (2016). Explaining the dynamics of the ultra-relativistic third Van Allen radiation belt. Nat. Phys., 12(10), 978-983. https://doi.org/10.1038/nphys3799

Mauk, B. H., Fox, N. J., Kanekal, S. G., Kessel, R. L., Sibeck, D. G., and Ukhorskiy, A. (2013). Science objectives and rationale for the radiation belt storm probes mission. Space Sci. Rev., 179(1-4), 3-27. https://doi.org/10.1007/s11214-0129908-y

Miyoshi, Y., Ono, T., Takashima, T., Asamura, K., Hirahara, M., Kasaba, Y. et al. (2015). The energization and radiation in geospace (ERG) project. Geophys. Monogr., 199, 103-116.

Olson, W. P., and Pfitzer, K. A. (1977). Magnetospheric magnetic field modeling. Technical Report, Huntington Beach, California: McDonnell Douglas Astronautics Co.

Selesnick, R. S., Su, Y. J., and Blake, J. B. (2016). Control of the innermost electron radiation belt by large-scale electric fields. J. Geophys. Res. Space Phys., 121(9), 8417-8427. https://doi.org/10.1002/2016JA022973

Tuszewski M., Cayton T. E., Ingraham J. C., et al. (2004). Bremsstrahlung effects 
in energetic particle detectors. Space Weather, 2, S10S01.

Violet, M. D., and Frederickson, A. R. (1993). Spacecraft anomalies on the CRRES satellite correlated with the environment and insulator samples. IEEE Trans. Nucl. Sci., 40(6), 1512-1520. https://doi.org/10.1109/23.273511

Wang, C. Q., Zhang, X., Zhang, L. G., Zhang, R. Y., Jin, L. Q., Sun, Y. Q., Jing, T., Zhang K. Y., Zhang, H. X., ... Chang, Z. (2017). Space radiation environment observations and applications based on GEO satellites. Aerosp. Shanghai (in Chinese), 34(4), 85-95. https://doi.org/10.19328/j.cnki.10061630.2017.04.011

Wang, L. H., Zong, Q. G., Shi, Q. Q., Wang, Y. F., Tu, C. Y., He, J. S., Tian, H., Wimmer-Schweingruber, R. F., and Zou, H. (2017). Discrete energetic ( 50-200 keV) electron events in the high-altitude cusp/polar cap/lobe. Sci. China Technol. Sci., 60(12), 1935-1940. https://doi.org/10.1007/s11431-0179119-x

Wilken, B., Axford, W. I., Daglis, I., Daly, P., Güttler, W., Ip, W. H., Korth, A., Kremser, G., Livi, S., ... Ullaland, S. (1997). RAPID-The imaging energetic particle spectrometer on cluster. Space Sci. Rev., 79(1-2), 399-473. https://doi.org/10.1023/A:1004994202296

Xiao, Z., Zou, J. Q., Zou, H., Zhong, W. Y., Huo, H. D., Bao, S. L., Xu, P. F., Zhu, W. M., and Wu, Z. X. (2003). Energetic particle detector on board ' $Z Y-1$ ' satellite. Acta Sci. Nat. Univ. Pekinensis (in Chinese), 39(3), 361-369. https://doi.org/10.3321/j.issn:0479-8023.2003.03.011

Yin, Z. F., Zou, H., Ye, Y. G., Zong, Q. G., and Wang, Y. F. (2019). Superposed epoch analysis of the energetic electron flux variations during CIRs measured by BD-IES. Space Wea., 17(12), 1765-1782. https://doi.org/10.1029/2019SW002296

Yu, X. Q., Song S. Y., Chen H. F., Qu, Y. N., Zou, H., Zong, Q. G., Shi, W. H., Zou, J. P., Zhong, W. Y., ... Shao, S. P. (2020). Monitoring deep dielectric charging effects in space. IEEE Trans. Nucl. Sci, 67(4), 716-721. https://doi.org/10.1109/TNS.2019.2963171

Zong, Q. G., Hao, Y. X., Zou, H., Fu, S. Y., Zhou, X. Z., Ren, J., Wang, L. H., Yuan, C. J., Liu, Z. Y., ... Wang, Y. F. (2016). Radial propagation of magnetospheric substorm-injected energetic electrons observed using a BD-IES instrument and Van Allen Probes. Sci. China Earth Sci., 59(7), 1508-1516. https://doi.org/10.1007/s11430-016-0039-0
Zong, Q. G., Wang, Y. F., Zou, H., Wang, L. H., Rankin, R., and Zhang, X. X. (2018). New magnetospheric substorm injection monitor: image electron spectrometer on board a Chinese navigation IGSO satellite. Space Wea., 16(2), 121-125. https://doi.org/10.1002/2017SW001708

Zou, H., Xiao, Z., Zou, J. Q., Zhong, W. Y., Hao, Y. Q., Zhang, D. H., Ma, Y. Q., Wang, H. Y., Xu, Y. P., ... Wu, Z. X. (2004). A comparison between detections of energetic electron by ZY1/CBMC and SZ2/XD. Chin. J. Geophys., 47(4), 644-651. https://doi.org/10.1002/cjg2.3532

Zou, H., Xiao, Z., Hao, Y. Q., Zou, J. Q., Zhu, W. M., Wu, Z. X., Xiang, H. W. (2006a). Analysis of the observation of particle detector inside 'CBERS-1' satellite under solar quiet conditions. Sci. China Ser. E, 49(3), 342-357. https://doi.org/10.1007/s11431-006-0342-9

Zou, H., Xiao, Z., Hao, Y. Q., Zou, J. Q., Zhong, W. Y., Wu, Z. X., Xiang, H. W., and Zhu, W. M. (2006b). Observation of the disturbed events by the particle detector inside "ZY-1" satellite. Chin. J. Geophys., 49(3), 559-564. https://doi.org/10.1002/cjg2.869

Zou, H., Chen, H. F., Zou, J. Q., Shi, W. H., Xiao, Z., Hao, Y. Q., Wu, Z. X., Xiang, H. W., and Zhu, W. M. (2007). Comparison between the observation of the particle detector inside ' $\mathrm{ZY}-1$ ' Satellite and the model of the radiation belt. Chin. J. Geophys., 50(3), 593-598. https://doi.org/10.1002/cjg2.1071

Zou, H., Luo, L., Li, C. F., Jia, X. H., Xu, F., Chen, H. F., Chen, J., Shi, W. H., Yu, X. Q., and Zou, J. Q. (2013). Angular response of 'pin-hole' imaging structure measured by collimated $\beta$ source. Sci. China Technol. Sci., 56(11), 2675-2680. https://doi.org/10.1007/s11431-013-5376-1

Zou, H., Ye, Y. G., Zong, Q. G., Chen, H. F., Zou, J. Q., Chen, J., Shi, W. H., Yu, X. Q., Zhong, W. Y., ... Zhang, X. X. (2018). Imaging energetic electron spectrometer on board a Chinese navigation satellite in the inclined GEO orbit. Sci. China Technol. Sci., 61(12), 1845-1865. https://doi.org/10.1007/s11431-018-9321-7

Zou, H., Ye, Y. G., Zong, Q. G., Chen, H. F., Luo, L., Zhou, X. Z., Chen, X. R., Hao, Y. X., Ren, J.... Zhang, X. X. (2019). Monte Carlo simulations of the sensor head of imaging energetic electron spectrometer on board a Chinese IGSO navigation satellite. Sci. China Technol. Sci., 62(7), 1169-1181. https://doi.org/10.1007/s11431-017-9314-6 